\title{
Stable Fractional Matchings ${ }^{\star}$
}

\author{
Ioannis Caragiannis ${ }^{\mathrm{a}}$, Aris Filos-Ratsikas ${ }^{\mathrm{b}}$, Panagiotis Kanellopoulos ${ }^{\mathrm{c}}$, Rohit Vaish ${ }^{\mathrm{d}, *}$ \\ ${ }^{a}$ Department of Computer Science, Aarhus University, Åbogade 34, 8200 Aarhus N, Denmark \\ ${ }^{b}$ Department of Computer Science, University of Liverpool, Liverpool, United Kingdom, L69 3BX \\ ${ }^{c}$ School of Computer Science and Electronic Engineering, University of Essex, Colchester, United Kingdom, CO4 $3 S Q$ \\ ${ }^{d}$ School of Technology and Computer Science, Tata Institute of Fundamental Research, Mumbai, India 400005
}

\begin{abstract}
We study a generalization of the classical stable matching problem that allows for cardinal preferences (as opposed to ordinal) and fractional matchings (as opposed to integral). In this cardinal setting, stable fractional matchings can have much larger social welfare than stable integral ones. Our goal is to understand the computational complexity of finding an optimal (i.e., welfare-maximizing) stable fractional matching. We consider both exact and approximate stability notions, and provide simple approximation algorithms with weak welfare guarantees. Our main result is that, somewhat surprisingly, achieving better approximations is computationally hard. To the best of our knowledge, these are the first computational complexity results for stable fractional matchings in the cardinal model. En route to these results, we provide a number of structural observations that could be of independent interest.
\end{abstract}

Keywords: Stable Matchings, Cardinal Preferences, Welfare Maximization

2010 MSC: 00-01, 99-00

\section{Introduction}

The stable matching problem is one of the most extensively studied problems at the interface of economics and computer science [1, 2, 3, 4, 5]. The input to the problem consists of the preference lists of two sets of agents, commonly referred to as the men and the women. The goal is to find a stable matching, i.e., a matching in which no pair of man and woman prefer each other over their assigned partners.

While the problem was originally motivated by college admissions [1], its applicability has subsequently expanded to various other domains such as medical residency [6, 7] and school choice [8]. In addition, the insights gained from the study of the stable matching problem, together with the development of computational tools and techniques in artificial intelligence, have shaped the design of modern two-sided matching

\footnotetext{
*A preliminary version of this article has appeared in the proceedings of the 2019 ACM Conference on Economics and Computation (pp. 21-39).

${ }^{*}$ Corresponding author

Email addresses: caragian@ceid.upatras.gr (Ioannis Caragiannis), arisfilosratsikas@gmail.com (Aris Filos-Ratsikas), panagiotis.kanellopoulos@essex.ac.uk (Panagiotis Kanellopoulos), rohit.vaish@tifr.res.in (Rohit Vaish)
} 

Gale-Shapley matchings, respectively [1]. The social welfare (i.e., the sum of utilities of all agents) of these matchings is $\mathcal{W}\left(\mu_{1}\right)=\mathcal{W}\left(\mu_{2}\right)=7$. 


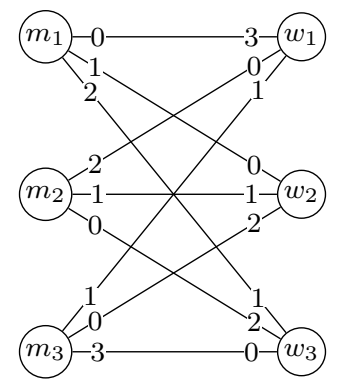

Figure 1: An instance with cardinal preferences.

Define $\mu_{3}:=\left\{\left(m_{1}, w_{1}\right),\left(m_{2}, w_{2}\right),\left(m_{3}, w_{3}\right)\right\}$, and notice that $\mathcal{W}\left(\mu_{3}\right)=8$. Now consider a fractional

We formalize the above question by defining the optimization problem Optimal STABLE Fractional Matching. To motivate this problem, we strengthen the observation in Example 1 to show that the social

\footnotetext{
${ }^{1}$ Observe that the gain in social welfare of $\mu$ was achieved by including an unstable integral matching $\mu_{3}$ in its support. One could consider alternative notions such as ex-post stability 11] wherein the support consists only of stable integral matchings. We discuss this and various other stability notions in Section 7 and find that insisting on a purely stable support results in significant welfare loss (Remark 3). In fact, there exists instances where the support of an optimal stable fractional matchings consists only of unstable integral matchings (Proposition 4 .
} 
welfare gap between the best stable fractional and the best stable integral matchings can be arbitrarily large. We show that the favorable welfare properties of stable fractional matchings come at the cost of limiting the algorithmic tools at our disposal. Specifically, we show that the set of stable fractional matchings can be non-convex (Proposition 3), and that, in the worst case, stable fractional matchings can have a large support (Theorem 3), thus prohibiting the use of support enumeration algorithms. Nevertheless, we present simple algorithms for Optimal Stable Fractional Matching with approximation ratio of $1+$ $\sigma_{\max } / \sigma_{\min }$, where $\sigma_{\max }$ and $\sigma_{\min }$ represent the maximum and minimum positive valuation in the input instance, respectively (Theorem 5). For the variant Optimal $\varepsilon$-Stable Fractional Matching, where the stability constraints are relaxed by a multiplicative factor of $(1-\varepsilon)$, an embarrassingly simple algorithm computes $1 / \varepsilon$-approximate solutions (Theorem 6). We then proceed to our main results (Theorems 7 and 8), which show that these approximation guarantees are - somewhat surprisingly — almost the best achievable via polynomial-time algorithms (unless $\mathrm{P}=\mathrm{NP}$ ). To the best of our knowledge, these are the first computational complexity results for stable fractional matchings in the cardinal preferences model.

The rest of the paper is organized as follows. We present related work in the matching literature in Section 1.2 We continue in Section 2 with preliminary definitions and warm up with exponential-time algorithms that solve Optimal Stable Fractional Matching using linear programming. Section 3 presents the structural properties of (nearly)-optimal solutions of Optimal Stable Fractional Matching. Our algorithms are presented in Section 4 and our inapproximability results are presented in Section 5 . Some proofs and additional material appear in the appendix.

\subsection{Related work}

The stable matching problem has been extensively studied for integral matchings. The universal existence of a stable integral matching [1] has led to considerable research on stable solutions that, in addition, optimize various measures of fairness or efficiency. A relevant example is that of optimizing the average rank of matched partners, which, in our model, corresponds to finding an optimal stable integral matching when the cardinal utilities are completely specified by the ordinal ranks (e.g., if an agent is ranked at position $i$, then it is valued at $n+1-i$ ). This problem is known to admit combinatorial polynomial-time algorithms [21, 24]. Other examples of such problems include minimizing the difference between average ranks of matched partners of men and women [25, optimizing the rank of matched partner for the least well-off agent [20, 26, 4], and maximizing (or minimizing) the cardinality of the matching [27, 23, 28].

A growing body of work in artificial intelligence and multiagent systems has studied variants of the stable matching problem motivated by practical considerations such as minimizing the amount of information exchange required in arriving at stable outcomes [29, 30] or ensuring strategyproofness in many-to-one matchings with quota constraints [31, 32. Various other papers have used computational approaches such as SAT solving [33, 34, 35] and constraint programming [36, 37, 38, in developing practically efficient solutions 
to computationally hard variants of the problem, as well as in axiomatic study of matching procedures [39.

Stability has also been studied in the context of fractional matchings. Starting with the works of Vande Vate [40, Rothblum [41, and Roth et al. [1], there is now a well-developed literature on linear programming

\footnotetext{
${ }^{2}$ The term "fractional stable matching" has been overloaded in the literature. For example, Teo and Sethuraman 22$]$ use it to refer to a feasible solution of stable matching linear program, and Aharoni and Fleiner 44] and Biró and Fleiner [45] use it in the study of hypergraphic preference systems to refer to a slightly different solution concept. We refer the reader to [46] for a detailed overview of these notions.

${ }^{3}$ The price of anarchy in this context is the worst-case multiplicative welfare gap between an optimal matching and an integral stable matching.
} 
for these models is an interesting avenue for future research.

\section{Preliminaries}

An instance of Stable Matching problem with Cardinal preferences (SMC) is given by the tuple $\langle M, W, U, V\rangle$, where $M:=\left\{m_{1}, \ldots, m_{n}\right\}$ and $W:=\left\{w_{1}, \ldots, w_{n}\right\}$ denote the set of $n$ men and $n$ women, respectively, and

$U$ and $V$ are $n \times n$ matrices of non-negative rational numbers that specify the valuations of the agents. Specifically, $U(m, w)$ is the value derived by man $m$ from his match with woman $w$, and $V(m, w)$ is the value derived by woman $w$ from her match with man $m$. Many of our results will focus on two special classes of valuations, namely binary (where $U, V \in\{0,1\}^{n \times n}$ ) and ternary valuations (where $U, V \in\{0,1, \alpha\}^{n \times n}$ for some $\alpha>1)$.

We will often describe an SMC instance using its graph representation. An instance $\mathcal{I}=\langle M, W, U, V\rangle$ can be represented as a bipartite graph with vertex sets $M$ and $W$, and an edge for every pair $(m, w) \in M \times W$ such that at least one of $U(m, w)>0$ or $V(m, w)>0$ holds. Each edge $(m, w)$ in this graph has two valuations associated with it, namely $U(m, w)$ and $V(m, w)$.

A fractional matching $\mu: M \times W \rightarrow \mathbb{R}_{\geq 0}$ is an assignment of non-negative weights to all man-woman pairs such that $\sum_{w \in W} \mu(m, w) \leq 1$ for each $m \in M$ and $\sum_{m \in M} \mu(m, w) \leq 1$ for each $w \in W$. A fractional matching $\mu$ is said to be complete if $\sum_{w \in W} \mu(m, w)=1$ for each man $m \in M$ and $\sum_{m \in M} \mu(m, w)=1$ for each woman $w \in W$. An integral matching $\mu$ is a fractional matching with weights $\mu(m, w) \in\{0,1\}$ for every pair $(m, w)$. With slight abuse of notation, we sometimes view an integral matching $\mu$ as a set of pairs and write $(m, w) \in \mu$ in place of $\mu(m, w)=1$. Also, unless stated otherwise, we will assume that any fractional/integral matching is complete.

It is well-known, and follows from the Birkhoff-von Neumann $(\mathrm{BvN})$ theorem, that a (complete) fractional matching $\mu$ can be written as a convex combination of $k=\mathcal{O}\left(n^{2}\right)$ integral matchings $\mu^{(1)}, \mu^{(2)}, \ldots, \mu^{(k)}$ so that for every pair $(m, w) \in M \times W$, we have

$$
\mu(m, w)=\sum_{j=1}^{k} \lambda_{j} \cdot \mu^{(j)}(m, w),
$$

where $\lambda_{j}>0$ for all $j \in\{1, \ldots, k\}$ and $\sum_{j=1}^{k} \lambda_{j}=1$. The set of integral matchings $\left\{\mu^{(1)}, \ldots, \mu^{(k)}\right\}$ is called the support of the fractional matching $\mu$. Note that the support need not be unique.

We proceed with the formal definitions of stability and approximate stability, which, in turn, depend on the utility derived by agents in a fractional matching. In particular, the utility derived by the man $m$ in $\mu$ is given by $u_{m}(\mu):=\sum_{w \in W} U(m, w) \mu(m, w)$, and the utility derived by the woman $w$ is given by $v_{w}(\mu):=\sum_{m \in M} V(m, w) \mu(m, w)$.

Definition 1 (Stability [19, 17]). Given a fractional matching $\mu$, a man-woman pair $(m, w)$ is said to be a blocking pair if $u_{m}(\mu)<U(m, w)$ and $v_{w}(\mu)<V(m, w)$. A fractional matching $\mu$ is stable if there are no 
blocking pairs, i.e., for each $(m, w) \in M \times W$, either $u_{m}(\mu) \geq U(m, w)$ or $v_{w}(\mu) \geq V(m, w)$.

Thus, under a stable fractional matching, no pair of man and woman can simultaneously improve by breaking away from the fractional matching and instead being integrally matched with each other. This notion of deviation is also reasonable from the viewpoint of bounded rationality, as agents only form blocking coalitions of size two, and only deviate to an integral matching between the members of the coalition.

Definition 2 ( $\varepsilon$-Stability [19]). Given any $\varepsilon \in[0,1)$ and a fractional matching $\mu$, a man-woman pair $(m, w)$ is said to be $\varepsilon$-blocking if $u_{m}(\mu)<(1-\varepsilon) U(m, w)$ and $v_{w}(\mu)<(1-\varepsilon) V(m, w)$; otherwise, the pair is said to be $\varepsilon$-stable. A fractional matching $\mu$ is $\varepsilon$-stable if all pairs are $\varepsilon$-stable.

Thus, a 0.01-stable fractional matching is one in which, for every man-woman pair, at least one of the two agents already receives (at least) $99 \%$ of the utility that he or she would receive by being integrally matched with the other. Note that a stable fractional matching is also $\varepsilon$-stable for every $\varepsilon \geq 0$.

Notice that Definitions 1 and 2 entail that agents in a blocking pair prefer to switch to an integral match with each other. One could also consider an alternative formulation wherein the agents merely prefer to increase their mutual fractional engagement, possibly at the expense of weakening other less preferred matches. This is precisely the notion of strong stability (see Section 7 for the definition) which has been studied in the context of ordinal preferences [11,46]. However, as we note in Remark 3 in Section 7, a strongly stable fractional matching can be strictly suboptimal in terms of social welfare, which further justifies the consideration of the stability notion in Definition 1 .

The next statement follows from the seminal result of Gale and Shapley [1].

Proposition 1. Given any SMC instance $\mathcal{I}$, a stable fractional matching $\mu$ for $\mathcal{I}$ always exists and can be computed in polynomial time.

Proposition 1] was originally proven in [1] in the standard stable matching model with ordinal preferences and integral matchings. It is easy to see that given any SMC instance $\mathcal{I}$, if an integral matching $\mu$ is stable for an ordinal instance derived from $\mathcal{I}$ (where the ordinal preferences of each agent are consistent with its valuations, breaking ties arbitrarily), then it is also stable for the original instance $\mathcal{I}$.

Next, we define social welfare, which is a measure of the efficiency of a fractional matching.

Definition 3 (Social welfare). Given an SMC instance $\langle M, W, U, V\rangle$ and a fractional matching $\mu$, the social welfare of $\mu$ is defined as

$$
\mathcal{W}(\mu):=\sum_{m \in M} u_{m}(\mu)+\sum_{w \in W} v_{w}(\mu)=\sum_{m \in M} \sum_{w \in W}(U(m, w)+V(m, w)) \mu(m, w) .
$$

An optimal matching is one with the highest social welfare among all fractional matchings. It follows from the $\mathrm{BvN}$ decomposition that an optimal matching is, without loss of generality, integral. Similarly, an 
optimal stable fractional matching (respectively, optimal $\varepsilon$-stable fractional matching) is one with the highest social welfare among all stable (respectively, all $\varepsilon$-stable) fractional matchings. We will use Optimal Stable Fractional Matching and Optimal $\varepsilon$-Stable Fractional Matching to refer to the corresponding optimization problems.

We will also discuss stable fractional matchings that are approximately optimal.

Definition 4 ( $\rho$-efficiency). For $\rho \in(0,1]$, the term $\rho$-efficient will refer to a stable (respectively, $\varepsilon$-stable) fractional matching with welfare at least $\rho$ times the welfare of the optimal stable (respectively, $\varepsilon$-stable) fractional matching. That is, $\mu$ is a $\rho$-efficient stable fractional matching if it is stable (respectively, $\varepsilon$-stable) and $\mathcal{W}(\mu) \geq \rho \mathcal{W}\left(\mu^{*}\right)$, where $\mu^{*}$ is an optimal stable (respectively, $\varepsilon$-stable) fractional matching.

Thus, an optimal stable (or $\varepsilon$-stable) fractional matching is 1-efficient.

\subsection{Computing optimal stable fractional matchings}

We will now discuss two exponential-time algorithms for Optimal Stable Fractional Matching. The first algorithm uses the following mixed integer linear program (OPT-Stab).

(OPT-Stab)

$$
\begin{aligned}
& \operatorname{maximize} \quad \sum_{m \in M} u_{m}+\sum_{w \in W} v_{w} \\
& \text { subject to } \quad u_{m} \geq U(m, w) y(m, w) \quad \forall m \in M, w \in W \\
& v_{w} \geq V(m, w)(1-y(m, w)) \quad \forall m \in M, w \in W \\
& u_{m}=\sum_{w \in W} U(m, w) \mu(m, w) \quad \forall m \in M \\
& v_{w}=\sum_{m \in M} V(m, w) \mu(m, w) \quad \forall w \in W \\
& \sum_{w \in W} \mu(m, w) \leq 1 \quad \forall m \in M \\
& \sum_{m \in M} \mu(m, w) \leq 1 \quad \forall w \in W \\
& \mu(m, w) \geq 0 \quad \forall m \in M, w \in W \\
& y(m, w) \in\{0,1\} \quad \forall m \in M, w \in W
\end{aligned}
$$

The non-negative weights $\mu(m, w)$ of man-woman pairs as well as the utilities $u_{m}:=u_{m}(\mu)$ and $v_{w}:=$ $v_{w}(\mu)$ of the agents (set in equalities (3) and (4) ) are the fractional variables of (OPT-Stab). The binary variables $y(m, w)$ encode the stability requirements for pair $(m, w)$ in constraints (1) and (2). Indeed, by setting $y(m, w)$ to 1 or 0 , we can require either $u_{m}(\mu) \geq U(m, w)$ or $v_{w}(\mu) \geq V(m, w)$. Constraints (5) and (6) ensure feasibility. By enumerating over all possible combinations of values for the binary variables $y(m, w)$ for $(m, w) \in M \times W$, we get $2^{n^{2}}$ different linear programs, and at least one of them must have the optimal stable fractional matching as its optimal solution. 
Our second algorithm is slightly faster and solves at most $\mathcal{O}\left(n^{n}\right)$ linear programs. It exploits the following linear program (OPT-Thresh)] which is defined using non-negative constants $\theta_{m}$ for $m \in M$ and $\theta_{w}$ for $w \in W$, which we call utility thresholds.

(OPT-Thresh)

$$
\begin{aligned}
& \operatorname{maximize} \quad \sum_{m \in M} u_{m}+\sum_{w \in W} v_{w} \\
& \text { subject to } u_{m} \geq \theta_{m} \quad \forall m \in M \\
& v_{w} \geq \theta_{w} \quad \forall w \in W \\
& u_{m}=\sum_{w \in W} U(m, w) \mu(m, w) \quad \forall m \in M \\
& v_{w}=\sum_{m \in M} V(m, w) \mu(m, w) \quad \forall w \in W \\
& \sum_{w \in W} \mu(m, w) \leq 1 \quad \forall m \in M \\
& \sum_{m \in M} \mu(m, w) \leq 1 \quad \forall w \in W \\
& \mu(m, w) \geq 0 \quad \forall m \in M, w \in W
\end{aligned}
$$

When all utility thresholds are set to zero, the solution of (OPT-Thresh) is an optimal (i.e., welfaremaximizing) fractional matching. Using (OPT-Thresh) to maximize social welfare under stability constraints is more challenging. We say that a set of utility thresholds is stability-preserving if for every pair of agents $m \in M$ and $w \in W$, either $\theta_{m} \geq U(m, w)$ or $\theta_{w} \geq V(m, w)$. Note that any fractional matching $\mu$ that is feasible for (OPT-Stab) is also feasible for (OPT-Thresh) for some stability-preserving set of utility thresholds (in particular, set the threshold of an agent equal to its utility under $\mu$ ). Conversely, any fractional matching $\mu$ that is feasible for (OPT-Thresh) with some set of stability-preserving utility thresholds is also feasible for (OPT-Stab).

One could now adopt the following strategy to solve Optimal Stable Fractional Matching: First, enumerate all $\mathcal{O}\left(n^{n}\right)$ tuples of utility thresholds $\left(\theta_{m_{1}}, \ldots, \theta_{m_{n}}\right)$ with $\theta_{m} \in\{U(m, w): w \in W\}$ for every man $m \in M$. Next, for every choice of $\left(\theta_{m_{1}}, \ldots, \theta_{m_{n}}\right)$, solve (OPT-Thresh) after appropriately setting $\left(\theta_{w_{1}}, \ldots, \theta_{w_{n}}\right)$ where $\theta_{w} \in\{V(m, w): m \in M\}$ for all $w \in W$, so that the set of utility thresholds is stability-preserving. Among these solutions, the fractional matching with highest social welfare will be the solution of Optimal Stable Fractional Matching. We note that (OPT-Thresh) resembles the integer programming formulations with cut-off variables in other works [53, 54].

\subsection{The case of symmetric valuations}

As mentioned previously in Section 1.2 a result by Deligkas et al. [47 implies NP-hardness of the problem of computing an optimal stable integral matching on SMC instances. The construction of Deligkas et al. involves a restricted class of SMC instances wherein the agents have symmetric (i.e., $U=V$ ) and ternary valuations in $\{0,1, \alpha\}$ with $\alpha \in(1,2)$. 
Proposition 2. Computing an optimal stable integral matching is NP-hard even for SMC instances with symmetric ternary valuations.

Our next result (Lemma 1) shows that for these SMC instances, the optimal stable fractional matching is, without loss of generality, integral. Consequently, the result in [47 implies NP-hardness of OptimaL Stable Fractional Matching.

Lemma 1. Let $\mathcal{I}$ be an SMC instance with symmetric and ternary valuations, and let $\mu^{*}$ be an optimal stable fractional matching for $\mathcal{I}$. Then, there exists a stable integral matching $\mu^{\mathrm{s}}$ such that $\mathcal{W}\left(\mu^{\mathrm{s}}\right)=\mathcal{W}\left(\mu^{*}\right)$.

Proof. Consider an optimal stable fractional matching $\mu^{*}$ that is not integral (i.e., has support of size at least two), and let $\mu$ be any integral matching in any support of $\mu^{*}$. We will show that $\mu$ is a stable integral matching. This would imply that all matchings in the support of $\mu^{*}$ are optimal stable integral matchings, as desired.

Assume, for contradiction, that $\mu$ is not stable, and let $(m, w)$ be a blocking pair in $\mu$. Then, because of symmetry, either $U(m, w)=V(m, w)=1$ or $U(m, w)=V(m, w)=\alpha$.

Suppose that $U(m, w)=V(m, w)=1$. Then, either both $m$ and $w$ are unmatched in $\mu$, or any pair $\left(m^{\prime}, w^{\prime}\right)$ in $\mu$ with either $m^{\prime}=m$ or $w^{\prime}=w$ satisfies $U\left(m^{\prime}, w^{\prime}\right)=V\left(m^{\prime}, w^{\prime}\right)=0$. By replacing such pairs with $(m, w)$ in $\mu$ (and, subsequently, in the support of $\mu^{*}$ ), we get a stable fractional matching with a higher welfare than $\mu^{*}$, contradicting its optimality.

Now suppose that $U(m, w)=V(m, w)=\alpha$. Then, either both $m$ and $w$ are unmatched in $\mu$, or any pair $\left(m^{\prime}, w^{\prime}\right)$ in $\mu$ with either $m^{\prime}=m$ or $w^{\prime}=w$ satisfies $U\left(m^{\prime}, w^{\prime}\right)=V\left(m^{\prime}, w^{\prime}\right) \in\{0,1\}$. However, since $\mu$ is in the support of $\mu^{*}$ (i.e., with strictly positive weight) and all valuations are less than or equal to $\alpha$, the utilities of both $m$ and $w$ in $\mu^{*}$ will be strictly smaller than $\alpha$, contradicting the stability of $\mu^{*}$.

From Lemma 1 and Proposition 2, we immediately have the following:

Corollary 1. Optimal Stable Fractional Matching is NP-hard.

\section{Structural properties}

In this section, we present several observations about the structure of optimal and nearly-optimal stable 245 fractional matchings. We begin by considerably strengthening our observation in Example 1 regarding the welfare gap between stable fractional and stable integral matchings.

Theorem 1. For every $\delta>0$ and $\alpha \geq 2$, there exists an SMC instance with ternary valuations in $\{0,1, \alpha\}$ and an optimal stable fractional matching $\mu^{*}$ such that any stable integral matching $\mu^{s}$ satisfies $\mathcal{W}\left(\mu^{s}\right) \leq$ $\left(\alpha-\frac{1}{2}-\delta\right)^{-1} \mathcal{W}\left(\mu^{*}\right)$ 


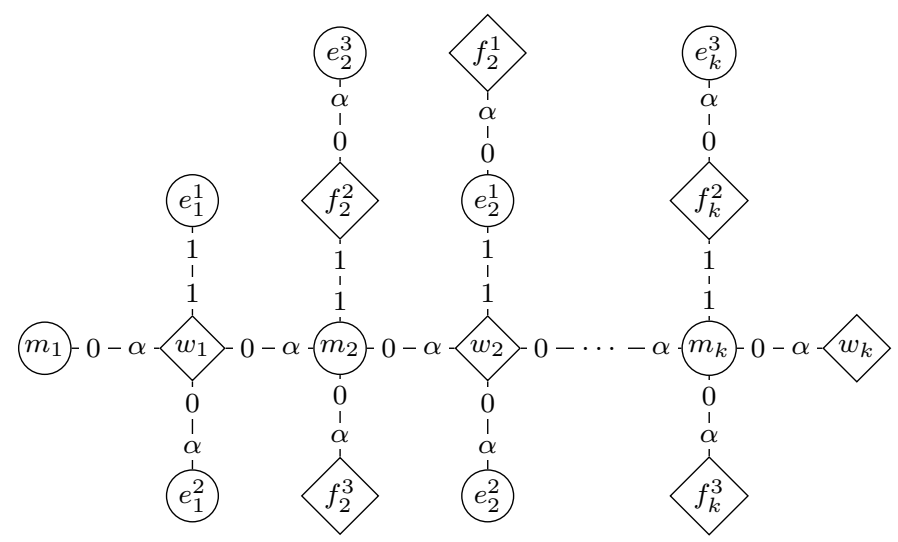

Figure 2: The SMC instance used in the proof of Theorem 1 As a convention, in graph representations where the two sides of the bipartition do not appear as left and right, we use circles to represent men and diamonds to represent women.

Proof. Consider the SMC instance shown in Figure 2, which, for some $k$ (to be determined later), consists of man $m_{i}$ and woman $w_{i}$ for all $i \in\{1, \ldots, k\}$, men $e_{i}^{1}$ and $e_{i}^{2}$ for all $i \in\{1, \ldots, k-1\}$, woman $f_{i}^{1}$ for all $i \in\{2, \ldots, k-1\}$, man $e_{i}^{3}$ and women $f_{i}^{2}$ and $f_{i}^{3}$ for all $i \in\{2, \ldots, k\}$.

To specify the valuations, we will use the following notation: For any $a, b \geq 0$, we will say that a manwoman pair $(m, w)$ is an " $a-b$ " edge if $U(m, w)=a$ and $V(m, w)=b$. In the instance in Figure 2 , the pairs $\left\{\left(m_{i}, w_{i}\right)\right\}_{i=1}^{k},\left\{\left(m_{i}, f_{i}^{3}\right)\right\}_{i=2}^{k}$, and $\left\{\left(e_{i}^{1}, f_{i}^{1}\right)\right\}_{i=2}^{k-1}$ are $0-\alpha$ edges, the pairs $\left\{\left(m_{i+1}, w_{i}\right)\right\}_{i=1}^{k-1},\left\{\left(e_{i}^{2}, w_{i}\right)\right\}_{i=1}^{k-1}$, and $\left\{\left(e_{i}^{3}, f_{i}^{2}\right)\right\}_{i=2}^{k}$ are $\alpha-0$ edges, and the pairs $\left\{\left(e_{i}^{1}, w_{i}\right)\right\}_{i=1}^{k-1}$ and $\left\{\left(m_{i}, f_{i}^{2}\right)\right\}_{i=2}^{k}$ are $1-1$ edges. All other pairs are $0-0$ edges. We remark that a slightly modified version of the instance in Figure 2 will be used again later in the proof of Theorem 7] (refer to the accumulator gadget in Figure 7a).

Consider a stable integral matching $\mu^{s}$. Since the pair $\left(e_{1}^{1}, w_{1}\right)$ is a $1-1$ edge, the stability requirement dictates that either $\left(m_{1}, w_{1}\right)$ or $\left(e_{1}^{1}, w_{1}\right)$ is contained in $\mu^{s}$. Either of these pairs contribute at most $\alpha$ to the social welfare (recall that $\alpha \geq 2$ ). Additionally, since $\mu^{s}$ is an integral matching, we get that $\mu^{s}\left(m_{2}, w_{1}\right)=0$. This, in turn, forces the pair $\left(m_{2}, f_{2}^{2}\right)$ to be contained in $\mu^{s}$ as well (or, otherwise, both $m_{2}$ and $f_{2}^{2}$ will have zero utility and violate stability). Continuing in this manner, we observe that the stability requirement for the pairs $\left\{\left(m_{i}, f_{i}^{2}\right)\right\}_{i=2}^{k}$ and $\left\{\left(e_{i}^{1}, w_{i}\right)\right\}_{i=2}^{k-1}$ will force these pairs to be contained in $\mu$ as well. Each of these pairs contributes 2 to the social welfare, and thus, $\mathcal{W}\left(\mu^{s}\right) \leq 4 k-6+\alpha$.

Now define a stable fractional matching $\mu$ as follows: Set $\mu\left(m_{i}, w_{i}\right)=1 / \alpha$ for all $i \in\{1, \ldots, k\}$, $\mu\left(e_{i}^{2}, w_{i}\right)=1-2 / \alpha, \mu\left(m_{i+1}, w_{i}\right)=1 / \alpha, \mu\left(e_{i}^{1}, w_{i}\right)=0$ for all $i \in\{1, \ldots, k-1\}, \mu\left(m_{i}, f_{i}^{2}\right)=0, \mu\left(m_{i}, f_{i}^{3}\right)=$ $1-2 / \alpha, \mu\left(e_{i}^{3}, f_{i}^{2}\right)=1$ for all $i \in\{2, \ldots, k\}$, and $\mu\left(e_{i}^{1}, f_{i}^{1}\right)=1$, for all $i \in\{2, \ldots, k-1\}$. (A similar matching will be used later in the proof of Lemma 5.) It is easy to verify that the social welfare of $\mu$ is $\mathcal{W}(\mu)=4 k(\alpha-1 / 2)-5 \alpha+3$. Furthermore, for each $i \in\{1, \ldots, k\}$, both man $m_{i}$ and woman $w_{i}$ have utility 1 in $\mu$ (i.e., $u_{m_{i}}(\mu)=v_{w_{i}}(\mu)=1$ ). Every $1-1$ edge includes one of these agents, and these are the only edges that need to be checked for stability (any other pair has at least one agent with a valuation of zero and, hence, 


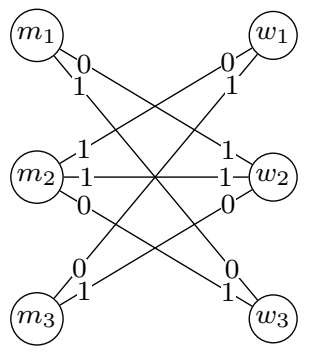

(a)

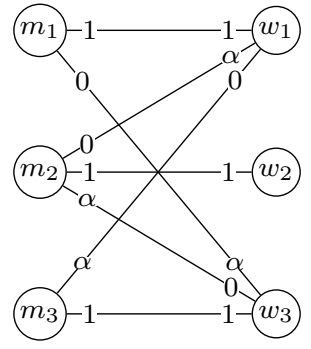

(b)

Figure 3: The SMC instances used in the proofs of Propositions 3 and 4

the stability constraint for those pairs is trivially satisfied). Therefore, $\mu$ is stable. The theorem follows by setting $k$ to be sufficiently large; specifically, for any given $\delta>0$, choosing $k \geq \frac{1}{4} \cdot\left(6-\alpha+\frac{\alpha^{2}-23 \alpha / 2+6}{\delta}\right)$ gives the desired bound.

We emphasize that Theorem 1 is a positive result as it establishes that stable fractional matchings can have much higher welfare than their integral counterparts, and highlights the importance of OpTIMAL Stable Fractional Matching.

Our next observation (Proposition 3) shows that the set of stable fractional matchings can be non-convex even for binary valuations. Interestingly, this does not prove to be a barrier in efficiently solving OptimaL Stable Fractional Matching in this setting (see Theorem 4 in Section 4 ).

Proposition 3. There exists an SMC instance with binary valuations for which the set of stable fractional matchings is non-convex.

Proof. Consider the instance $\mathcal{I}=\langle M, W, U, V\rangle$ with three men $m_{1}, m_{2}, m_{3}$ and three women $w_{1}, w_{2}, w_{3}$, whose graph representation and agent valuations are shown in Figure $3 \mathrm{a}$. Consider the integral matchings $\mu^{(1)}:=\left\{\left(m_{1}, w_{3}\right),\left(m_{2}, w_{1}\right),\left(m_{3}, w_{2}\right)\right\}$ and $\mu^{(2)}:=\left\{\left(m_{1}, w_{2}\right),\left(m_{2}, w_{3}\right),\left(m_{3}, w_{1}\right)\right\}$. It is easy to verify that both $\mu^{(1)}$ and $\mu^{(2)}$ are stable for $\mathcal{I}$. However, the fractional matching $\mu:=0.5 \mu^{(1)}+0.5 \mu^{(2)}$ is not stable since $\left(m_{2}, w_{2}\right)$ is a blocking pair; indeed, $0.5=u_{m_{2}}(\mu)<U\left(m_{2}, w_{2}\right)=1$ and $0.5=v_{w_{2}}(\mu)<V\left(m_{2}, w_{2}\right)=1$.

Remark 1. A follow-up work to the conference version of our paper has shown that non-convexity also holds for strict preferences [55].

Remark 2. It is worth comparing the non-convexity results in Proposition 3 and Remark 1 with similar results for other stability notions. In particular, Aziz and Klaus [46. Theorem 1] have shown that the set of strongly stable matchings (see Section 7 for the definition) can be non-convex. In Section 8 , we revisit the counterexample used in their proof, which is stated in terms of ordinal preferences, and show that there exists a realization of cardinal preferences consistent with their instance such that the relevant convex combination 
of (strongly) stable matchings is still stable. Thus, the non-convexity result of Aziz and Klaus [46] for strong stability does not imply the same for the set of stable matchings.

The structure of stable fractional matchings becomes even more interesting (and, as we will see in Section 5 also computationally unwieldy) when we move to ternary valuations. It turns out that the

300 Indeed, the utilities of the agents in $\mu$ are given by $u_{m_{1}}(\mu)=0, u_{m_{2}}(\mu)=1, u_{m_{3}}(\mu)=\alpha-1, v_{w_{1}}(\mu)=1$, $v_{w_{2}}(\mu)=\frac{\alpha-2}{\alpha-1}$ and $v_{w_{3}}(\mu)=\alpha-\frac{1}{\alpha-1}$. Notice that only the pairs $\left(m_{1}, w_{1}\right),\left(m_{2}, w_{2}\right)$, and $\left(m_{3}, w_{3}\right)$ need to be checked for stability. For each of these pairs, at least one of the agents has a utility of at least 1 in $\mu$, implying that $\mu$ is stable. Finally, notice that $\mu\left(m_{1}, w_{1}\right)=0$, which means that the unique stable integral support of a stable fractional matching can comprise entirely of unstable integral matchings (Proposition 4), and its size can grow linearly with the input (Theorem 3). These observations pose major limitations on the set of algorithmic tools at our disposal.

Proposition 4. There exists an SMC instance with ternary valuations and a stable fractional matching $\mu$ such that every integral matching in any support of $\mu$ is unstable.

Proof. Consider the SMC instance $\mathcal{I}=\langle M, W, U, V\rangle$ with three men and three women shown in Figure $3 \mathrm{~b}$. The parameter $\alpha \geq 3$ is a constant. There are six different perfect integral matchings:

- Matching $\mu^{(1)}$, which consists of pairs $\left(m_{1}, w_{1}\right),\left(m_{2}, w_{2}\right)$, and $\left(m_{3}, w_{3}\right)$ and has a social welfare of 6 . It is easy to verify that this is the unique stable integral matching. Also, any subset of $\mu^{(1)}$ is not stable as the pair that is missing from $\mu^{(1)}$ will be blocking.

- Matching $\mu^{(2)}$, which consists of pairs $\left(m_{1}, w_{2}\right),\left(m_{2}, w_{3}\right)$, and $\left(m_{3}, w_{1}\right)$ and has a social welfare of $2 \alpha$. The matching is not stable since the pair $\left(m_{1}, w_{1}\right)$ is blocking.

- Matching $\mu^{(3)}$, which consists of pairs $\left(m_{1}, w_{3}\right),\left(m_{2}, w_{1}\right)$, and $\left(m_{3}, w_{2}\right)$ and has a social welfare of $2 \alpha$. It is not stable since $\left(m_{2}, w_{2}\right)$ is blocking.

- Matching $\mu^{(4)}$, which consists of pairs $\left(m_{1}, w_{1}\right),\left(m_{2}, w_{3}\right)$, and $\left(m_{3}, w_{2}\right)$ and has a social welfare of $\alpha+2$. It is not stable since $\left(m_{3}, w_{3}\right)$ is blocking.

- Matching $\mu^{(5)}$, which consists of pairs $\left(m_{1}, w_{3}\right),\left(m_{2}, w_{2}\right)$, and $\left(m_{3}, w_{1}\right)$ and has a social welfare of $2 \alpha+2$. It is not stable since $\left(m_{1}, w_{1}\right)$ is blocking.

- Matching $\mu^{(6)}$, which consists of pairs $\left(m_{1}, w_{2}\right),\left(m_{2}, w_{1}\right)$, and $\left(m_{3}, w_{3}\right)$ and has a social welfare of $\alpha+2$. It is not stable since the pair $\left(m_{2}, w_{2}\right)$ is blocking.

Consider the matching $\mu:=\frac{1}{\alpha(\alpha-1)} \cdot \mu^{(2)}+\frac{1}{\alpha} \cdot \mu^{(3)}+\frac{\alpha-2}{\alpha-1} \cdot \mu^{(5)}$. It is easy to verify that $\mu$ is stable. matching $\mu^{(1)}$ cannot occur in any support of $\mu$. 
We remark that with some extra work, one can show that the matching $\mu$ in the proof of Proposition 4 is the unique optimal stable fractional matching.

One might wonder whether the support of an optimal stable fractional matching always consists of an optimal integral matching. This turns out to not be the case, as illustrated by the following instance: Consider two men $m_{1}, m_{2}$ and two women $w_{1}, w_{2}$ with valuations given by $U=\left[\begin{array}{ll}2 & 0 \\ 1 & 0\end{array}\right]$ and $V=\left[\begin{array}{ll}0 & 0 \\ 1 & 2\end{array}\right]$. The unique optimal integral matching is $\left\{\left(m_{1}, w_{1}\right),\left(m_{2}, w_{2}\right)\right\}$ while the unique optimal stable fractional matching is $\left\{\left(m_{1}, w_{2}\right),\left(m_{2}, w_{1}\right)\right\}$.

As mentioned previously in Section 2 , a (stable) fractional matching is the convex combination of at most $n^{2}$ integral ones. Theorem 2 provides a stronger bound on the support size of an optimal stable fractional matching.

Theorem 2. Given any SMC instance $\mathcal{I}$, there exists an optimal stable fractional matching for $\mathcal{I}$ with at most $4 n$ integral matchings in its support.

Proof. Let $\mu^{*}$ be an optimal stable fractional matching for $\mathcal{I}$. Recall from Section 2.1 that $\mu^{*}$ solves the program (OPT-Thresh) for some set of stability-preserving utility thresholds. Observe that (OPT-Thresh) has $n^{2}$ free variables (we ignore here the $2 n$ variables $u_{m}$ for $m \in M$ and $v_{w}$ for $w \in W$, which depend on the remaining ones according to constraints (11) and (12). Without loss of generality, $\mu^{*}$ is an optimal extreme point solution of (OPT-Thresh). That is, when (OPT-Thresh) is instantiated for $\mu^{*}, n^{2}$ linearly independent inequality constraints become tight. Among them, at most $4 n$ can correspond to the sets of constraints (9), 10), (13), and (14). The remaining ones must correspond to the set of constraints (15), implying that at least $n^{2}-4 n$ free variables will be equal to zero. Thus, $\mu^{*}$ can assign positive weights to at most $4 n$ man-woman pairs and, consequently, can have at most $4 n$ integral matchings in its support.

Next we show that the bound in Theorem 2 is tight up to a constant factor.

Theorem 3. For every $\rho \in(0,1]$, there exists a family of SMC instances with ternary valuations for which any support of a $\rho$-efficient stable fractional matching consists of $\Omega(\rho n)$ integral matchings.

Proof. Consider a family of SMC instances $\mathcal{I}_{n}=\langle M, W, U, V\rangle$ with $M=\left\{m_{1}, \ldots, m_{n}\right\}$ and $W=\left\{w_{1}, \ldots, w_{n}\right\}$, where $n$ is odd. Let $\alpha$ be such that $\alpha>\max \left\{n+2, \frac{2 n}{\rho(n-1)}\right\}$. The (ternary) valuations of the agents are defined as follows: For each $i \in\{1,2, \ldots, n\}, U\left(m_{i}, w_{i}\right)=V\left(m_{i}, w_{i}\right)=1$. For each $i \in\left\{1,2, \ldots, \frac{n-1}{2}\right\}$, $U\left(m_{2 i}, w_{1}\right)=U\left(m_{2 i+1}, w_{2 i}\right)=V\left(m_{2 i}, w_{2 i+1}\right)=\alpha$ and $V\left(m_{2 i}, w_{1}\right)=V\left(m_{2 i+1}, w_{2 i}\right)=U\left(m_{2 i}, w_{2 i+1}\right)=0$. Finally, $U\left(m_{n}, w_{1}\right)=0$ and $V\left(m_{n}, w_{1}\right)=\alpha$. For all remaining pairs $(m, w) \in M \times W, U(m, w)=V(m, w)=$ 0. Figure $4 a$ illustrates the SMC instance $\mathcal{I}_{5}$.

Define $\mu^{\mathrm{opt}}:=\left\{\left(m_{1}, w_{1}\right)\right\} \cup\left\{\left(m_{2 i}, w_{2 i+1}\right),\left(m_{2 i+1}, w_{2 i}\right): i \in\left\{1,2, \ldots, \frac{n-1}{2}\right\}\right\}$ (see Figure 40 ). We also define a number of other integral matchings obtained by modifying $\mu^{\mathrm{opt}}$, as follows: For all $i \in\left\{1,2, \ldots, \frac{n-1}{2}\right\}$, 


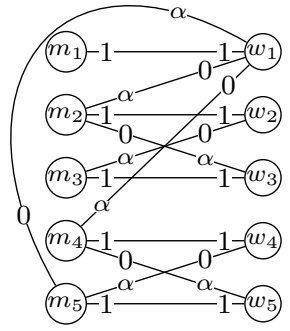

(a)

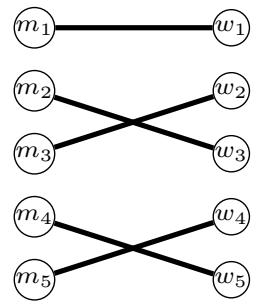

(b)

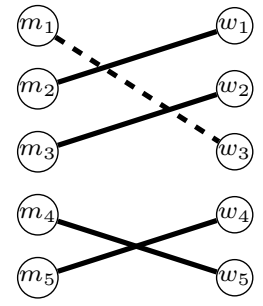

(c)

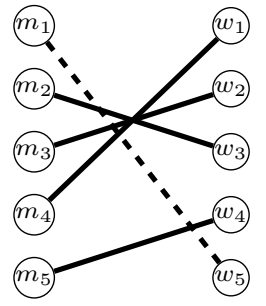

(d)

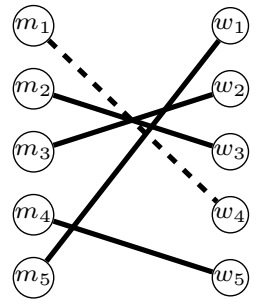

(e)

Figure 4: Subfigure (a) illustrates the graph representation of the SMC instance $\mathcal{I}_{n}$ described in the proof of Theorem 3 for $n=5$. Subfigure (b) shows the matching $\mu^{\mathrm{opt}}$. Subfigures (c), (d), and (e) show the matchings $\mu^{(1)}, \mu^{(2)}$, and $\mu^{(3)}$, respectively. Dashed lines indicate zero-valuation pairs that do not appear in the graph representation.

the matching $\mu^{(i)}$ (see Figures 4 and $4 \mathrm{~d}$ ) is the integral matching which is obtained from $\mu^{\text {opt }}$ by replacing $\left\{\left(m_{1}, w_{1}\right),\left(m_{2 i}, w_{2 i+1}\right)\right\}$ with $\left\{\left(m_{1}, w_{2 i+1}\right),\left(m_{2 i}, w_{1}\right)\right\}$, i.e.,

$$
\mu^{(i)}:=\left\{\left(m_{1}, w_{2 i+1}\right) \cup\left(m_{2 i}, w_{1}\right) \cup\left(m_{2 i+1}, w_{2 i}\right) \cup\left\{\left(m_{2 \ell}, w_{2 \ell+1}\right) \cup\left(m_{2 \ell+1}, w_{2 \ell}\right)\right\}_{\ell \in\left\{1,2, \ldots, \frac{n-1}{2}\right\} \backslash\{i\}}\right\} .
$$

Also, the matching $\mu^{\left(\frac{n+1}{2}\right)}$ (see Figure 4 ) is the integral matching obtained from $\mu^{\text {opt }}$ by replacing $\left\{\left(m_{1}, w_{1}\right),\left(m_{n}, w_{n-1}\right)\right\}$ with $\left\{\left(m_{1}, w_{n-1}\right),\left(m_{n}, w_{1}\right)\right\}$, i.e.,

$$
\mu^{\left(\frac{n+1}{2}\right)}:=\left\{\left(m_{1}, w_{n-1}\right) \cup\left(m_{n-1}, w_{n}\right) \cup\left(m_{n}, w_{1}\right) \cup\left\{\left(m_{2 \ell}, w_{2 \ell+1}\right) \cup\left(m_{2 \ell+1}, w_{2 \ell}\right)\right\}_{\ell \in\left\{1,2, \ldots, \frac{n-3}{2}\right\}}\right\} .
$$

Now, consider the fractional matching $\mu:=\sum_{i=1}^{\frac{n+1}{2}} \frac{1}{\alpha} \mu^{(i)}+\left(1-\frac{n+1}{2 \alpha}\right) \mu^{\text {opt }}$. Since $\alpha>n+2>\frac{n+1}{2}, \mu$ is well-defined and has the matchings $\mu^{\mathrm{opt}}$ and $\mu^{(i)}$ for all $i \in\left\{1, \ldots, \frac{n+1}{2}\right\}$ in its support. Notice that $\mathcal{W}\left(\mu^{\mathrm{opt}}\right)=(n-1) \alpha+2$ and $\mathcal{W}\left(\mu^{(i)}\right)=(n-1) \alpha$ for all $i \in\left\{1,2, \ldots, \frac{n+1}{2}\right\}$. Thus, $\mathcal{W}(\mu)>(n-1) \alpha$.

It can be verified that $\mu$ is stable. Indeed, we only need to check the blocking condition for the pairs $\left(m_{i}, w_{i}\right)$ with $i \in\{1,2, \ldots, n\}$. We have that $v_{w_{1}}(\mu) \geq 1$ (since $\mu^{\left(\frac{n+1}{2}\right)}$ has weight $\frac{1}{\alpha}$ in $\mu$ and $\left.V\left(m_{n}, w_{1}\right)=\alpha\right)$, $u_{m_{2 i}}(\mu) \geq 1$ for each $i \in\left\{1,2, \ldots, \frac{n-1}{2}\right\}$ (since $\mu^{(i)}$ has weight $\frac{1}{\alpha}$ in $\mu$ and $U\left(m_{2 i}, w_{1}\right)=\alpha$ ), and $v_{w_{2 i+1}}(\mu) \geq 1$ for each $i \in\left\{1,2, \ldots, \frac{n-1}{2}\right\}$ (since $\mu^{(i)}$ has weight $\frac{1}{\alpha}$ in $\mu$ and $V\left(m_{2 i}, w_{2 i+1}\right)=\alpha$ ). The welfare of the optimal stable fractional matching must therefore be at least $\mathcal{W}(\mu)$, and thus strictly greater than $(n-1) \alpha$.

We now claim that any $\rho$-efficient stable fractional matching $\mu^{\prime}$ satisfies $\mu^{\prime}\left(m_{n}, w_{1}\right)>0$. Indeed, assuming otherwise that $\mu^{\prime}\left(m_{n}, w_{1}\right)=0$, the only pair that can give positive utility to man $m_{1}$ and woman $w_{1}$ is $\left(m_{1}, w_{1}\right)$. Hence, we must also have $\mu^{\prime}\left(m_{1}, w_{1}\right)=1$, and, as a result, $\mu^{\prime}\left(m_{2 i}, w_{1}\right)=0$ for all $i \in$ $\left\{1,2, \ldots, \frac{n-1}{2}\right\}$. Then, the only pair that can give positive utility to man $m_{2 i}$ and woman $w_{2 i}$ is $\left(m_{2 i}, w_{2 i}\right)$, and hence, it must also be that $\mu^{\prime}\left(m_{2 i}, w_{2 i}\right)=1$. Consequently, the only pair that can give positive utility to man $m_{2 i+1}$ and woman $w_{2 i+1}$ is $\left(m_{2 i+1}, w_{2 i+1}\right)$ and, hence, we must have $\mu^{\prime}\left(m_{2 i+1}, w_{2 i+1}\right)=1$. The welfare of matching $\mu^{\prime}$ would then be $2 n$, which is less than $\rho(n-1) \alpha$ by the assumed bound on $\alpha$. In other words, 
the welfare of $\mu^{\prime}$ would be less than $\rho$ times the welfare of the stable fractional matching $\mu$, contradicting the assumption that $\mu^{\prime}$ is $\rho$-efficient.

The final step in the proof involves showing that for any stable fractional matching $\mu^{\prime}$ with support of size at most $\frac{n-1}{2} \rho$, we must have $\mathcal{W}\left(\mu^{\prime}\right)<\rho(n-1) \alpha$; the desired bound on the support size would then follow from the contrapositive. Let $T:=\left\{i \in\left\{1,2, \ldots \frac{n-1}{2}\right\}: \mu^{\prime}\left(m_{2 i}, w_{1}\right)>0\right\}$, and $\bar{T}:=\left\{1,2, \ldots \frac{n-1}{2}\right\} \backslash T$. Since $\mu^{\prime}$ has support of size at most $\frac{n-1}{2} \rho$ and $\mu^{\prime}\left(m_{n}, w_{1}\right)>0$, it holds that $|T| \leq \frac{n-1}{2} \rho-1$.

For every $i \in T$, the agents $m_{2 i}, w_{2 i}, m_{2 i+1}$, and $w_{2 i+1}$ can together contribute at most $2 \alpha$ to the welfare. On the other hand, when $i \in \bar{T}$, we have $\mu^{\prime}\left(m_{2 i}, w_{1}\right)=0$, and the only pair that can give positive utility to man $m_{2 i}$ and woman $w_{2 i}$ is $\left(m_{2 i}, w_{2 i}\right)$. Therefore, we must have that $\mu^{\prime}\left(m_{2 i}, w_{2 i}\right)=1$. Consequently, the only pair that can give positive utility to man $m_{2 i+1}$ and woman $w_{2 i+1}$ is $\left(m_{2 i+1}, w_{2 i+1}\right)$, and it follows that $\mu^{\prime}\left(m_{2 i+1}, w_{2 i+1}\right)=1$. Therefore, when $i \in \bar{T}$, the agents $m_{2 i}, w_{2 i}, m_{2 i+1}$, and $w_{2 i+1}$ can together contribute at most 4 to the welfare. Taking the possible contribution of pair $\left(m_{1}, w_{1}\right)$ into account, we have that

$$
\begin{aligned}
\mathcal{W}\left(\mu^{\prime}\right) & \leq 2+2 \alpha|T|+4|\bar{T}|=2 n+(2 \alpha-4)|T| \\
& \leq 2 n+\rho(n-1) \alpha-2 \alpha-2(n-1) \rho+4<\rho(n-1) \alpha .
\end{aligned}
$$

The equality follows from the definition of $\bar{T}$, the second inequality follows from the bound on $|T|$ above, and the third one from the definition of $\alpha$. This completes the proof of Theorem 3 .

Theorem 3 has an interesting algorithmic implication. The fact that the support size can be large even for approximately optimal solutions suggests that a support enumeration strategy - which has proven useful in other economic problems [56, 57] - will be strictly more demanding than any of the MILP-based approaches in Section 2.1. Specifically, a brute force enumeration of all subsets of $\Omega(\rho n)$ matchings requires $\Omega\left((n !)^{\rho n}\right) \approx$ $\Omega\left(n^{\rho n^{2}}\right)$ time. By contrast, the MILPs (OPT-Stab) and (OPT-Thresh) described in Section 2.1 require $\mathcal{O}\left(2^{n^{2}}\right)$ and $\mathcal{O}\left(n^{n}\right)$ time, respectively. A similar comparison can be made for optimal $\varepsilon$-stable fractional matchings. Interestingly, as we will show in Section 4 an $\varepsilon$-stable fractional matching of small support can be easily computed, and provides nearly the best approximation ratios achievable by efficient algorithms (under standard complexity-theoretic assumptions).

\section{Algorithmic results}

We begin the discussion of our algorithmic results with binary valuations. In this setting, OptimaL Stable Fractional Matching reduces to computing a maximum weight matching on a specific weighted graph associated with the given instance.

Theorem 4. Given an SMC instance $\mathcal{I}=\langle M, W, U, V\rangle$ with binary valuations, an optimal stable fractional matching for $\mathcal{I}$ is, without loss of generality, integral, and can be computed in polynomial time. 
Proof. Let $G$ be the graph representation of $\mathcal{I}$. We assign to each edge $(m, w)$ in $G$ a weight $\gamma(m, w)$, as follows:

$$
\gamma(m, w)= \begin{cases}2+\frac{1}{n^{2}} & \text { if } U(m, w)=V(m, w)=1 \\ 1 & \text { if } U(m, w)=1, V(m, w)=0 \text { or } U(m, w)=0, V(m, w)=1 \\ 0 & \text { otherwise. }\end{cases}
$$

Thus, for any integral matching $\mu$ in $G$, if $n_{\mu}$ denotes the number of agents (men and women) with utility 1 in the SMC instance $\mathcal{I}$, then $n_{\mu} \leq \sum_{(m, w) \in \mu} \gamma(m, w)<n_{\mu}+1$.

Let $\mu$ be a maximum weight matching in $G$. Note that $\mu$ can be computed in polynomial time and is, without loss of generality, integral. Also, it follows from the above inequality that $\mu$ is an optimal matching for $\mathcal{I}$. We will now argue that $\mu$ is stable. Indeed, assuming otherwise, any blocking pair $(m, w)$ must have $0=u_{m}(\mu)<U(m, w)=1,0=v_{w}(\mu)<V(m, w)=1$ and $(m, w) \notin \mu$. Thus, if $\mu$ contains one or both of the edges $\left(m, w^{\prime}\right)$ and $\left(m^{\prime}, w\right)$ for some $w^{\prime} \neq w$ and $m^{\prime} \neq m$, then we must have that $U\left(m, w^{\prime}\right)=0$ and/or $V\left(m^{\prime}, w\right)=0$. By our definition of weights, this would imply that $\gamma\left(m, w^{\prime}\right)=1$ and/or $\gamma\left(m^{\prime}, w\right)=1$. We can now replace one or both of these edges with the edge $(m, w)$, which has weight $\gamma(m, w)=2+1 / n^{2}$, and obtain a new matching with strictly larger weight - a contradiction.

The proof of Theorem 4 can be easily extended to the non-bipartite setting (also known as the stable roommates problem) to compute an optimal stable fractional matching with binary valuations.

Next, we consider general valuations and show how to exploit stable integral matchings to get an approximate solution for Optimal Stable Fractional Matching. Let $\sigma_{\max }$ and $\sigma_{\min }$ denote the largest and the smallest non-zero valuation among all agents in $\mathcal{I}$, respectively. We call a man-woman pair $(m, w)$ light if either $U(m, w)=0$ or $V(m, w)=0$, and heavy otherwise. Given an SMC instance $\mathcal{I}$ as input, our algorithm computes a stable integral matching for $\mathcal{I}$, say $\mu$, in two steps: First, it computes a stable integral matching $\mu_{1}$ using only the heavy pairs (and taking into account the stability constraints in heavy pairs only). Then, it completes the solution with a matching $\mu_{2}$ of maximum welfare using the light pairs subject to feasibility constraints, i.e., using light pairs that do not share any agents with the pairs in $\mu_{1}$. The light pairs impose no additional constraints on stability, so the resulting matching is stable.

We will show that $\mu$ has approximation ratio $1+\sigma_{\max } / \sigma_{\min }$ (recall that $\sigma_{\max }$ and $\sigma_{\min }$ are the largest and the smallest non-zero valuations, respectively, in the instance $\mathcal{I}$ ). Let $\mu^{\mathrm{opt}}$ be an optimal matching for $\mathcal{I}$. Also, let $\mu_{1}^{\mathrm{opt}}$ be the set of pairs of $\mu^{\mathrm{opt}}$ that share an agent with some pair of $\mu_{1}$, i.e., $\mu_{1}^{\mathrm{opt}}:=\{(m, w) \in$ $\mu^{\text {opt }}$ : at least one of $m$ or $w$ is matched in $\left.\mu_{1}\right\}$. By definition of $\mu_{2}$, we have $\mathcal{W}\left(\mu_{2}\right) \geq \mathcal{W}\left(\mu^{\text {opt }} \backslash \mu_{1}^{\text {opt }}\right)$. To complete the proof, we will need the following lemma.

Lemma 2. $\mathcal{W}\left(\mu_{1} \backslash \mu_{1}^{o p t}\right) \geq\left(1+\sigma_{\max } / \sigma_{\min }\right)^{-1} \mathcal{W}\left(\mu_{1}^{o p t} \backslash \mu_{1}\right)$.

Proof. Our proof constructs a mapping in which every pair $(m, w) \in \mu_{1}^{\mathrm{opt}} \backslash \mu_{1}$ is mapped to one of its agents, 
whom we will call the witness of the pair. The mapping is such that the utility of the witness in the matching $\mu_{1} \backslash \mu_{1}^{\text {opt }}$ is at least $\left(1+\sigma_{\max } / \sigma_{\min }\right)^{-1}(U(m, w)+V(m, w))$. Note that once we establish the said mapping, the proof will follow, since each agent can be the witness of at most one pair of $\mu_{1}^{\text {opt }} \backslash \mu_{1}$ and $\mathcal{W}\left(\mu_{1} \backslash \mu_{1}^{\text {opt }}\right)$ is at least the total utility of the witnesses in $\mu_{1} \backslash \mu_{1}^{\mathrm{opt}}$.

Consider a light pair $(m, w) \in \mu_{1}^{\mathrm{opt}} \backslash \mu_{1}$. The witness is an agent ( $m$ or $w$ ) who also belongs to a pair of $\mu_{1} \backslash$ $\mu_{1}^{\text {opt }}$; such an agent certainly exists by the definition of $\mu_{1}^{\text {opt }}$. Since all pairs of $\mu_{1} \backslash \mu_{1}^{\text {opt }}$ are heavy, the utility of the witness of $(m, w)$ in $\mu_{1} \backslash \mu_{1}^{\text {opt }}$ is at least $\sigma_{\min }=\frac{\sigma_{\min }}{\sigma_{\max }}\left(0+\sigma_{\max }\right) \geq\left(1+\sigma_{\max } / \sigma_{\min }\right)^{-1}(U(m, w)+V(m, w))$, since $(m, w)$ is light.

Now consider a heavy pair $(m, w) \in \mu_{1}^{\text {opt }} \backslash \mu_{1}$. If $\mu_{1}$ contains a pair $\left(m, w^{\prime}\right)$ with $U\left(m, w^{\prime}\right) \geq U(m, w)$, select agent $m$ to be the witness, otherwise select agent $w$. Note that in the latter case, stability of $\mu_{1}$ implies the existence of $\left(m^{\prime}, w\right) \in \mu_{1}$ such that $V\left(m^{\prime}, w\right) \geq V(m, w)$. Hence, the utility of the witness of $(m, w)$ in $\mu_{1} \backslash \mu_{1}^{\mathrm{opt}}$ is at least $\min \{U(m, w), V(m, w)\}$, which, in turn, is at least $\left(1+\sigma_{\max } / \sigma_{\min }\right)^{-1}(U(m, w)+V(m, w))$.

Now, Lemma 2 gives the desired approximation ratio, as follows:

$$
\begin{aligned}
\mathcal{W}(\mu) & =\mathcal{W}\left(\mu_{1}\right)+\mathcal{W}\left(\mu_{2}\right)=\mathcal{W}\left(\mu_{1} \backslash \mu_{1}^{\mathrm{opt}}\right)+\mathcal{W}\left(\mu_{1} \cap \mu_{1}^{\mathrm{opt}}\right)+\mathcal{W}\left(\mu_{2}\right) \\
& \geq\left(1+\sigma_{\max } / \sigma_{\min }\right)^{-1} \mathcal{W}\left(\mu_{1}^{\mathrm{opt}} \backslash \mu_{1}\right)+\mathcal{W}\left(\mu_{1}^{\mathrm{opt}} \cap \mu_{1}\right)+\mathcal{W}\left(\mu^{\mathrm{opt}} \backslash \mu_{1}^{\mathrm{opt}}\right) \\
& \geq\left(1+\sigma_{\max } / \sigma_{\min }\right)^{-1} \mathcal{W}\left(\mu^{\mathrm{opt}}\right)
\end{aligned}
$$

For ternary valuations in $\{0,1, \alpha\}$, the above algorithm gives a $(1+\alpha)$-approximation. An improved approximation for ternary valuations can be achieved using the following modification: When computing the stable integral matching, resolve ties in favour of the pairs $(m, w)$ with the highest $U(m, w)+V(m, w)$. The next lemma establishes an improved approximation ratio of $\max \{2, \alpha\}$.

Lemma 3. The modified algorithm for SMC instances with ternary valuations in $\{0,1, \alpha\}$ satisfies $\mathcal{W}\left(\mu_{1} \backslash\right.$ $\left.\mu_{1}^{o p t}\right) \geq \min \left\{\frac{1}{2}, \frac{1}{\alpha}\right\} \mathcal{W}\left(\mu_{1}^{o p t} \backslash \mu_{1}\right)$.

${ }_{440}$ Proof. For a pair $(m, w)$ of matching $\mu_{1}^{\text {opt }} \backslash \mu_{1}$, we use the term neighborhood to refer to the pairs of $\mu_{1} \backslash \mu_{1}^{\text {opt }}$ that use agent $m$ or $w$. We will show that the total utility from pairs in the neighborhood of $(m, w)$ is at least $\min \{1,2 / \alpha\}(U(m, w)+V(m, w))$. Since each pair of $\mu_{1} \backslash \mu_{1}^{\text {opt }}$ can be in the neighborhood of at most two pairs of $\mu_{1}^{\mathrm{opt}} \backslash \mu_{1}$, this will give us the desired inequality.

Indeed, by the particular way we resolve ties in the ordinal preferences before computing the matching 445 $\mu_{1}$, a heavy pair $(m, w)$ in $\mu_{1}^{\text {opt }} \backslash \mu_{1}$ must have a pair of utility at least $U(m, w)+V(m, w)$ in its neighborhood. A light pair $(m, w)$ has $U(m, w)+V(m, w) \leq \alpha$ and certainly has a heavy pair of utility at least 2 in its neighborhood.

The above discussion is summarized in the following statement. 
Theorem 5. There is a polynomial-time algorithm which, given an SMC instance $\mathcal{I}$ with an optimal matchin $\{0,1, \alpha\}$, and $\mathcal{W}(\mu) \geq\left(1+\sigma_{\max } / \sigma_{\min }\right)^{-1} \mathcal{W}\left(\mu^{\text {opt }}\right)$ in general, where $\sigma_{\max }$ and $\sigma_{\min }$ denote the highest and lowest non-zero valuation in $\mathcal{I}$, respectively.

We conclude this section by considering approximate stability. For general valuations, we present a polynomial-time $1 / \varepsilon$-approximation algorithm for Optimal $\varepsilon$-STABle Fractional MATChing, which constructs an $\varepsilon$-stable fractional matching with a small support by combining an optimal matching with a stable integral matching.

Theorem 6. There is a polynomial-time algorithm that given any $\mathrm{SMC}$ instance $\mathcal{I}=\langle M, W, U, V\rangle$ and any rational $\varepsilon \in[0,1]$, computes a fractional matching $\mu$ that is $\varepsilon$-stable for $\mathcal{I}$ such that $\mathcal{W}(\mu) \geq \varepsilon \mathcal{W}\left(\mu^{\text {opt }}\right)$, where $\mu^{\text {opt }}$ is an optimal matching for $\mathcal{I}$.

Proof. Let $\mu^{\mathrm{s}}$ be any stable integral matching and $\mu^{\text {opt }}$ be an optimal matching for $\mathcal{I}$. Note that both $\mu^{\mathrm{s}}$ and $\mu^{\mathrm{opt}}$ can be computed in polynomial time. We will show that $\mu:=(1-\varepsilon) \mu^{\mathrm{s}}+\varepsilon \mu^{\mathrm{opt}}$ satisfies the desired properties. Indeed, $\mathcal{W}(\mu)=(1-\varepsilon) \mathcal{W}\left(\mu^{\mathrm{s}}\right)+\varepsilon \mathcal{W}\left(\mu^{\mathrm{opt}}\right) \geq \varepsilon \mathcal{W}\left(\mu^{\mathrm{opt}}\right)$. Furthermore, since $\mu^{\mathrm{s}}$ is stable, we have that for any man-woman pair $(m, w) \in M \times W$, either $u_{m}\left(\mu^{\mathrm{s}}\right) \geq U(m, w)$ or $v_{w}\left(\mu^{\mathrm{s}}\right) \geq V(m, w)$. The former condition implies that $u_{m}(\mu) \geq(1-\varepsilon) u_{m}\left(\mu^{\mathrm{s}}\right) \geq(1-\varepsilon) U(m, w)$, while the latter condition gives $v_{w}(\mu) \geq(1-\varepsilon) V(m, w)$. Either way, the pair $(m, w)$ is $\varepsilon$-stable.

In particular, Theorem 6 shows that a $\frac{1}{2}$-stable fractional matching with welfare at least half of that of an optimal fractional matching (and therefore, that of an optimal stable fractional matching) can be computed in polynomial time. In Section 10, we provide a slightly stronger welfare guarantee: There is a polynomial-time algorithm that computes a $\frac{1}{2}$-stable fractional matching with welfare at least that of an optimal (exactly) stable fractional matching.

\section{Hardness of approximation}

In this section, we present our inapproximability statements, which are by far the technically most involved results in the paper. We present polynomial-time reductions which, given a 3SAT formula $\phi$ of a particular structure, construct SMC instances that simulate the evaluation of $\phi$ for every variable assignment. evaluation of $\phi$ by the SMC instance is such that:

(a) when $\phi$ has a satisfying assignment, there is a stable (or $\varepsilon$-stable) fractional matching where the contribution of the agents in the accumulator gadget to the welfare can be large and dominates the contribution from the remaining SMC instance, and 
(b) when $\phi$ is not satisfiable, the contribution of the accumulator and, subsequently, the total welfare of any stable (or $\varepsilon$-stable) fractional matching is very small.

Hence, distinguishing between SMC instances with stable (or $\varepsilon$-stable) fractional matchings of very high and very low welfare would allow us to decide 3SAT. We have two inapproximability statements: Theorem 7 for Optimal Stable Fractional Matching and Theorem 8 for Optimal $\varepsilon$-Stable Fractional MATChing.

Theorem 7. For every constant $\delta>0$, it is NP-hard to approximate Optimal Stable Fractional MATCHING for SMC instances with ternary valuations in $\{0,1, \alpha\}$ to within a factor of (i) $\alpha-1 / 2-\delta$ if $\alpha=\mathcal{O}(n)$, and (ii) $\Omega\left(n^{1-\delta}\right)$ otherwise.

Theorem 8. For any constant $\varepsilon \in(0,0.03]$ and $\delta>0$, it is NP-hard to approximate Optimal $\varepsilon$-Stable Fractional Matching to within a factor of $1 / \varepsilon-\delta$.

We will prove Theorem 7 here; the proof of Theorem 8 , which uses similar gadgets but is slightly more involved, appears in Section 9. Since the proof is long, we have divided it into three parts: the description of the reduction (Section 5.1), technical claims with gadget properties (Section 5.2), and the proof of the inapproximability result (Section 5.3).

\subsection{The reduction}

In particular, we present a polynomial-time reduction from 2P2N-3SAT, the special case of 3SAT consisting of 3-CNF clauses in which every variable appears four times: twice as a positive literal and twice as a negative one. 2P2N-3SAT is known to be NP-hard [58. Our reduction takes as input an instance of $2 \mathrm{P} 2 \mathrm{~N}-3 \mathrm{SAT}$ consisting of $N$ (boolean) variables $x_{1}, x_{2}, \ldots, x_{N}$, and a 3 -CNF formula $\phi$ with $L=4 N / 3$ clauses $c_{1}, c_{2}, \ldots, c_{L}$. Without loss of generality, we assume that each clause in $\phi$ consists of distinct literals.

Given the instance of $2 \mathrm{P} 2 \mathrm{~N}-3 \mathrm{SAT}$, our reduction generates an instance $\mathcal{I}=\langle M, W, U, V\rangle$ of OptIMAL Stable Fractional Matching. As usual, we denote by $n$ the number of men (or women) in $\mathcal{I}$. We will use a positive integer parameter $k$ which will determine the size of $n$; in particular, $n=\mathcal{O}(N+k)$. We define $\mathcal{I}$ by referring to its graph representation, which consists of variable gadgets, clause gadgets, variable-clause connectors, an accumulator, and clause-accumulator connectors. For each gadget, we classify the edges (i.e., man-woman pairs and their valuations) into the following three types:

- $m a n$-heavy edges $(m, w)$ with $U(m, w)=\alpha$ and $V(m, w)=0$,

- woman-heavy edges $(m, w)$ with $U(m, w)=0$ and $V(m, w)=\alpha$, and

- balanced edges $(m, w)$ with $U(m, w)=V(m, w)=1$. 


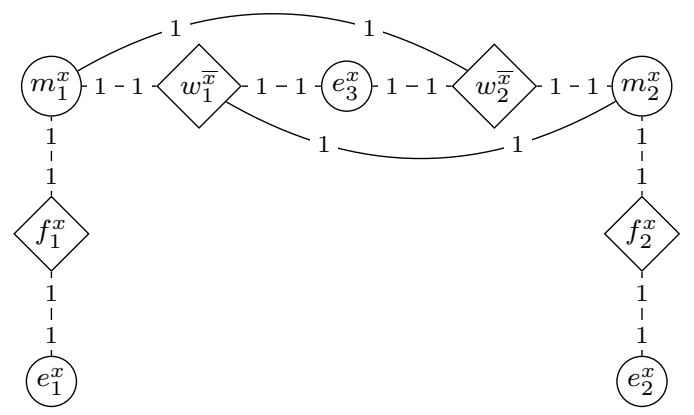

(a)

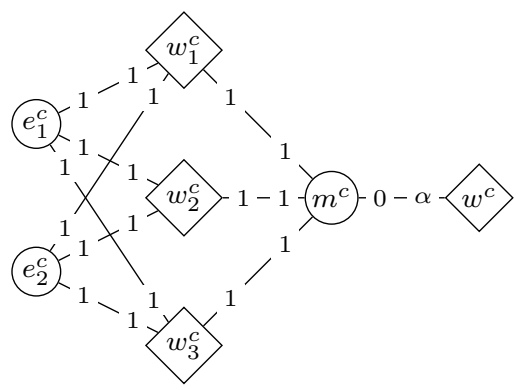

(b)

Figure 5: (a) The variable gadget corresponding to the variable $x$. (b) The clause gadget corresponding to the clause $c$ and its CA-connector $\left(m^{c}, w^{c}\right)$. $V(m, w)=0$.

The instance $\mathcal{I}$ has a variable gadget for every variable $x$, which consists of five men $m_{1}^{x}, m_{2}^{x}, e_{1}^{x}, e_{2}^{x}$, $e_{3}^{x}$, four women $w_{1}^{\bar{x}}, w_{2}^{\bar{x}}, f_{1}^{x}, f_{2}^{x}$ and the ten balanced edges $\left(e_{1}^{x}, f_{1}^{x}\right),\left(m_{1}^{x}, f_{1}^{x}\right),\left(m_{1}^{x}, w_{1}^{\bar{x}}\right),\left(e_{3}^{x}, w_{1}^{\bar{x}}\right),\left(e_{3}^{x}, w_{2}^{\bar{x}}\right)$, $\left(m_{2}^{x}, w_{2}^{\bar{x}}\right),\left(m_{2}^{x}, f_{2}^{x}\right),\left(e_{2}^{x}, f_{2}^{x}\right),\left(m_{1}^{x}, w_{2}^{\bar{x}}\right)$, and $\left(m_{2}^{x}, w_{1}^{\bar{x}}\right)$, as shown in Figure 5 a.

For every clause $c$, instance $\mathcal{I}$ has a clause gadget with three men $m^{c}, e_{1}^{c}, e_{2}^{c}$, three women $w_{1}^{c}, w_{2}^{c}, w_{3}^{c}$, and the nine balanced edges between them, as shown in Figure $5 \mathrm{~b}$.

For every appearance of a literal in a clause, there is a variable-clause connector (or VC-connector). The structure of VC-connectors depends on whether they correspond to positive or negative literals and the value of $\alpha$. In each case, we identify one edge of the $\mathrm{VC}$-connector as the input, and either one or two edges as the output.

Specifically, for every positive literal $x$ whose $i$-th appearance $(i \in\{1,2\})$ is as the $j$-th literal $(j \in$ $\{1,2,3\})$ of clause $c, \mathcal{I}$ has a VC-connector defined as follows:

- When $\alpha \geq 2$, the VC-connector consists of a single woman-heavy edge between $m_{i}^{x}$ (from the variable gadget corresponding to variable $x$ ) and $w_{j}^{c}$ (from the clause gadget corresponding to clause $c$ ), as shown in Figure 6a. This edge is simultaneously the input and the output edge of the VC-connector.

- When $\alpha \in(3 / 2,2)$, the VC-connector consists of woman $w^{x, c}$, man $m^{x, c}$, the woman-heavy edges $\left(m_{i}^{x}, w^{x, c}\right)$ and $\left(m^{x, c}, w_{j}^{c}\right)$, and the balanced edge $\left(m^{x, c}, w^{x, c}\right)$, as shown in Figure 6b. Here, $\left(m_{i}^{x}, w^{x, c}\right)$ is the input and $\left(m^{x, c}, w_{j}^{c}\right)$ is the output edge.

For every negative literal $\bar{x}$ whose $i$-th appearance $(i \in\{1,2\})$ is as the $j$-th literal $(j \in\{1,2,3\})$ of clause $c, \mathcal{I}$ has a VC-connector defined as follows:

- When $\alpha \geq 2$, the VC-connector consists of man $m^{\bar{x}, c}$, woman $w^{\bar{x}, c}$, the man-heavy edge $\left(m^{\bar{x}, c}, w_{i}^{\bar{x}}\right)$, 


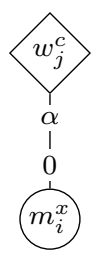

(a)

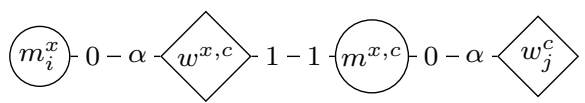

(b)

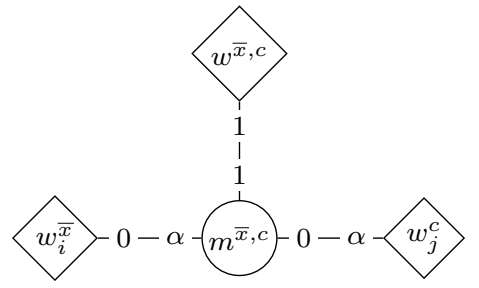

(c)

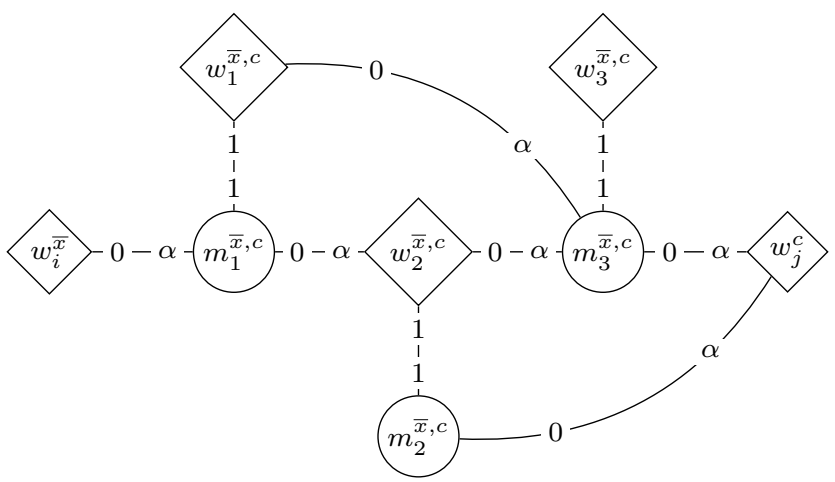

(d)

Figure 6: VC-connectors corresponding to clause $c$ and positive literal $x$ for (a) $\alpha \geq 2$ and (b) $\alpha \in(3 / 2,2)$, and to clause $c$ and negative literal $\bar{x}$ for (c) $\alpha \geq 2$ and (d) $\alpha \in(3 / 2,2)$.

the balanced edge $\left(m^{\bar{x}, c}, w^{\bar{x}, c}\right)$, and the woman-heavy edge $\left(m^{\bar{x}, c}, w_{j}^{c}\right)$, as shown in Figure 66. Here, $\left(m^{\bar{x}, c}, w_{i}^{\bar{x}}\right)$ is the input and $\left(m^{\bar{x}, c}, w_{j}^{c}\right)$ is the output edge.

- When $\alpha \in(3 / 2,2)$, the VC-connector consists of three men $m_{1}^{\bar{x}, c}, m_{2}^{\bar{x}, c}, m_{3}^{\bar{x}, c}$, three women $w_{1}^{\bar{x}, c}, w_{2}^{\bar{x}, c}$, $w_{3}^{\bar{x}, c}$, the man-heavy edges $\left(m_{1}^{\bar{x}, c}, w_{i}^{\bar{x}}\right),\left(m_{3}^{\bar{x}, c}, w_{1}^{\bar{x}, c}\right),\left(m_{3}^{\bar{x}, c}, w_{2}^{\bar{x}, c}\right)$, the woman-heavy edges $\left(m_{1}^{\bar{x}, c}, w_{2}^{\bar{x}, c}\right)$, $\left(m_{2}^{\bar{x}, c}, w_{j}^{c}\right),\left(m_{3}^{\bar{x}, c}, w_{j}^{c}\right)$, and the balanced edges $\left(m_{1}^{\bar{x}, c}, w_{1}^{\bar{x}, c}\right),\left(m_{2}^{\bar{x}, c}, w_{2}^{\bar{x}, c}\right),\left(m_{3}^{\bar{x}, c}, w_{3}^{\bar{x}, c}\right)$, as shown in Figure 6 6 d. In this case, the VC-connector has one input edge $\left(m_{1}^{\bar{x}, c}, w_{i}^{\bar{x}}\right)$ and two output edges $\left(m_{2}^{\bar{x}, c}, w_{j}^{c}\right)$ and $\left(m_{3}^{\bar{x}, c}, w_{j}^{c}\right)$.

The accumulator (Figure 7) of instance $\mathcal{I}$ has different structure depending on the value of $\alpha$. Its size depends on the positive integer parameter $k$.

- When $\alpha \geq 2$ (see Figure $7 \mathrm{a}$ ), the accumulator consists of man $m_{i}$ and woman $w_{i}$ for all $i \in\{1, \ldots, k\}$, men $e_{i}^{1}$ and $e_{i}^{2}$ and woman $f_{i}^{1}$ for all $i \in\{1, \ldots, k-1\}$, man $e_{i}^{3}$ and women $f_{i}^{2}$ and $f_{i}^{3}$ for all $i \in\{2, \ldots, k\}$, and woman $w^{c}$ for every clause $c$ of $\phi$. The edges comprise of man-heavy edges $\left(m_{i}, w_{i-1}\right)$ and $\left(e_{i}^{3}, f_{i}^{2}\right)$ for all $i \in\{2, \ldots, k\}$ and $\left(e_{i}^{2}, w_{i}\right)$ for all $i \in\{1, \ldots, k-1\}$, balanced edges $\left(m_{1}, w^{c}\right)$ for every clause $c$, which we call tine edges, $\left(e_{i}^{1}, w_{i}\right)$ for all $i \in\{1, \ldots, k-1\}$, and $\left(m_{i}, f_{i}^{2}\right)$ for all $i \in\{2, \ldots, k\}$, and woman-heavy edges $\left(m_{i}, w_{i}\right)$ for all $i \in\{1, \ldots, k\},\left(e_{i}^{1}, f_{i}^{1}\right)$ for all $i \in\{1, \ldots, k-1\}$, and $\left(m_{i}, f_{i}^{3}\right)$ for all $i \in\{2, \ldots, k\}$. 


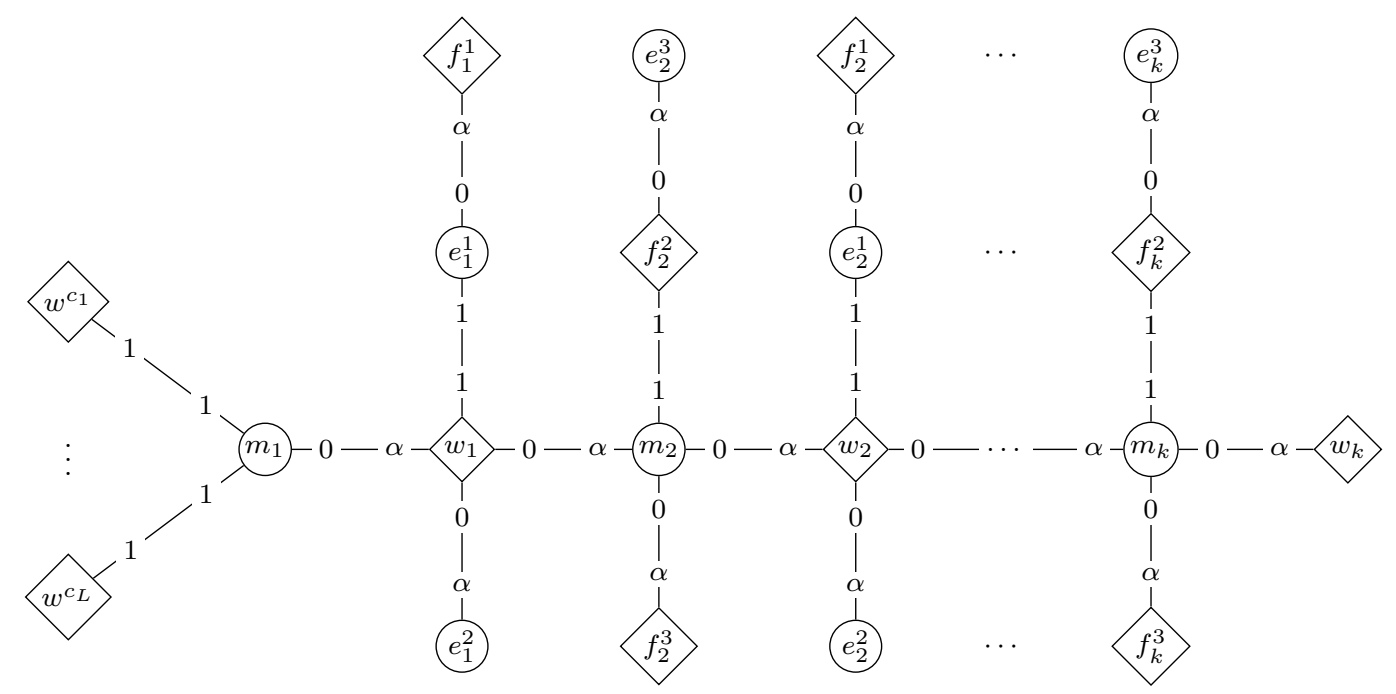

(a)

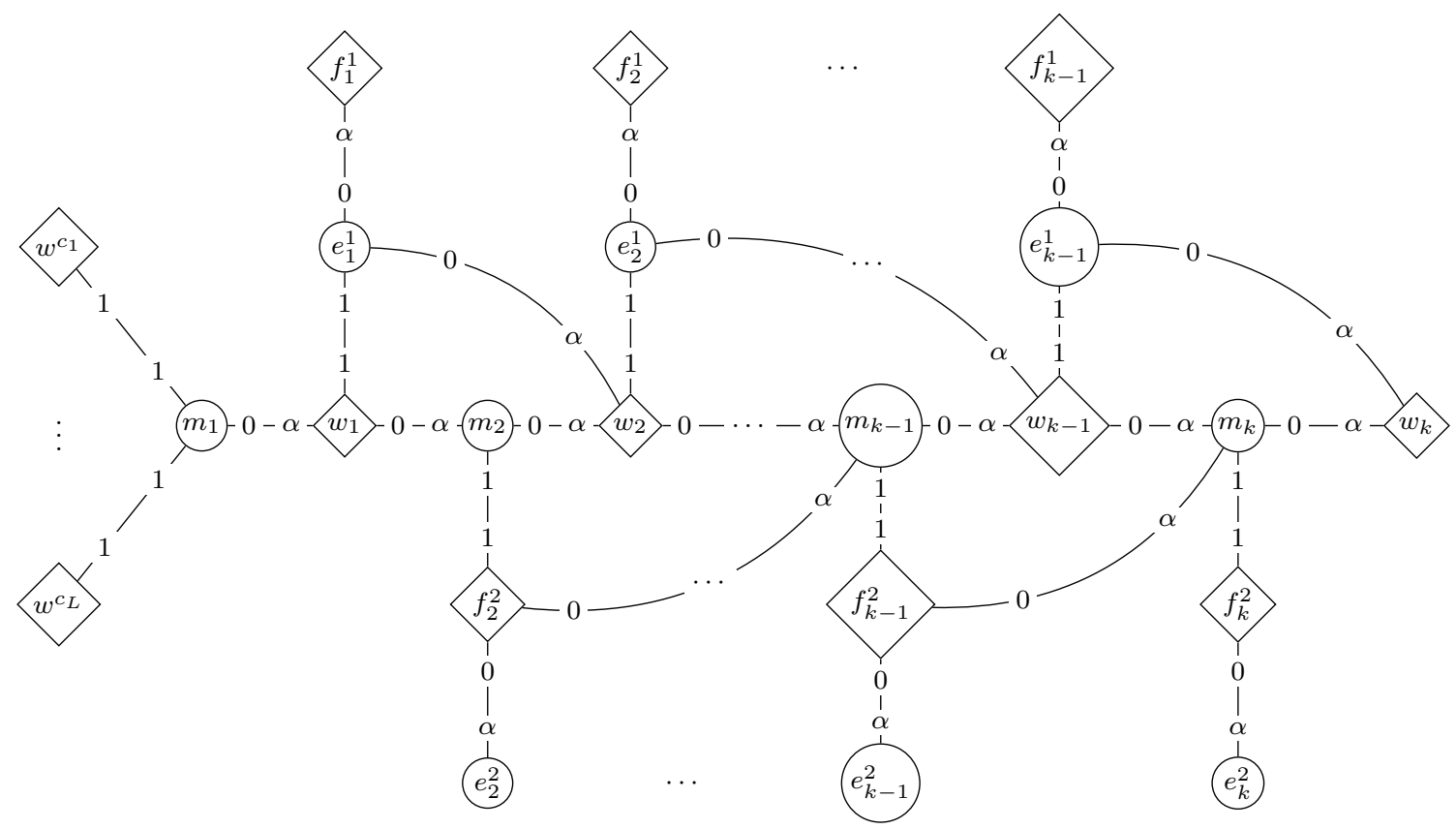

(b)

Figure 7: The accumulator for the cases (a) $\alpha \geq 2$ and (b) $\alpha \in(3 / 2,2)$. 
- When $\alpha \in(3 / 2,2)$ (see Figure $7 \mathrm{p})$, the accumulator consists of man $m_{i}$, woman $w_{i}$ for all $i \in\{1, \ldots, k\}$, man $e_{i}^{1}$ and woman $f_{i}^{1}$ for all $i \in\{1, \ldots, k-1\}$, man $e_{i}^{2}$ and woman $f_{i}^{2}$ for all $i \in\{2, \ldots, k\}$, and woman $w^{c}$ for every clause $c$ of $\phi$. The edges comprise of man-heavy edges $\left(m_{i}, w_{i-1}\right)$ and $\left(e_{i}^{2}, f_{i}^{2}\right)$ for all $i \in\{2, \ldots, k\}$ and $\left(m_{i}, f_{i-1}^{2}\right)$ for all $i \in\{3, \ldots, k\}$, the balanced edges $\left(m_{1}, w^{c}\right)$ for every clause $c$ (tine edges), $\left(e_{i}^{1}, w_{i}\right)$ for all $i \in\{1, \ldots, k-1\}$ and $\left(m_{i}, f_{i}^{2}\right)$ for all $i \in\{2, \ldots, k\}$, and the woman-heavy edges $\left(m_{i}, w_{i}\right)$ for all $i \in\{1, \ldots, k\}$, and $\left(e_{i}^{1}, f_{i}^{1}\right)$ and $\left(e_{i}^{1}, w_{i+1}\right)$ for all $i \in\{1, \ldots, k-1\}$.

Finally, instance $\mathcal{I}$ has a clause-accumulator connector (or $C A$-connector) for every clause $c$ of $\phi$ consisting of the woman-heavy edge $\left(m^{c}, w^{c}\right)$ between the man $m^{c}$ (from the clause gadget corresponding to clause $c$ ) and woman $w^{c}$ (from the accumulator); see Figure $5 \mathrm{~b}$. Notice that the above construction has more women than men. To restore balance, we pad the instance with extra (isolated) men that neither value nor are valued by any other agent. This completes the construction of the reduced instance.

\subsection{Gadget properties}

We will now prove several important properties (Claims 1, 4 ) of our construction.

Claim 1. For every variable $x$, a stable fractional matching $\mu$ satisfies at least one of the following:

(1) $\mu\left(m_{1}^{x}, w_{1}^{\bar{x}}\right)+\mu\left(m_{1}^{x}, w_{2}^{\bar{x}}\right)+\mu\left(m_{1}^{x}, f_{1}^{x}\right)=1$ and $\mu\left(m_{2}^{x}, w_{1}^{\bar{x}}\right)+\mu\left(m_{2}^{x}, w_{2}^{\bar{x}}\right)+\mu\left(m_{2}^{x}, f_{2}^{x}\right)=1$.

(2) $\mu\left(m_{1}^{x}, w_{1}^{\bar{x}}\right)+\mu\left(m_{2}^{x}, w_{1}^{\bar{x}}\right)+\mu\left(e_{3}^{x}, w_{1}^{\bar{x}}\right)=1$ and $\mu\left(m_{1}^{x}, w_{2}^{\bar{x}}\right)+\mu\left(m_{2}^{x}, w_{2}^{\bar{x}}\right)+\mu\left(e_{3}^{x}, w_{2}^{\bar{x}}\right)=1$.

Proof. Suppose, for contradiction, that for some $i, j \in\{1,2\}$, we have $\mu\left(m_{i}^{x}, w_{1}^{\bar{x}}\right)+\mu\left(m_{i}^{x}, w_{2}^{\bar{x}}\right)+\mu\left(m_{i}^{x}, f_{i}^{x}\right)<1$ and $\mu\left(m_{1}^{x}, w_{j}^{\bar{x}}\right)+\mu\left(m_{2}^{x}, w_{j}^{\bar{x}}\right)+\mu\left(e_{3}^{x}, w_{j}^{\bar{x}}\right)<1$. Then, both $m_{i}^{x}$ and $w_{j}^{\bar{x}}$ will have utility strictly less than 1 in $\mu$, and thus the pair $\left(m_{i}^{x}, w_{j}^{\bar{x}}\right)$ would be blocking.

We remark that the two conditions in the statement of Claim 1 affect the weight of the input edges of the VC-connectors that are attached to the variable gadget in any stable fractional matching. In particular, condition (1) implies that the weight assigned to the input edges of the VC-connectors that correspond to the two appearances of the positive literal $x$ in clauses must be 0 . To see why, observe that these input edges are incident to nodes $m_{1}^{x}$ and $m_{2}^{x}$, and the total weight of all edges incident to each of these nodes cannot exceed 1. Condition (2) has a similar implication for the edges associated with the negative literal $\bar{x}$.

Claim 2. Any stable fractional matching that assigns a weight of 0 to the input edge of a VC-connector must assign a weight of 0 to its output edge(s) as well.

Proof. For $\alpha \geq 2$, the claim holds trivially for VC-connectors corresponding to positive literals (Figure6a). Consider a VC-connector corresponding to a negative literal $\bar{x}$ and a clause $c$ containing it (Figure 6r). Observe that, besides the input edge $\left(m^{\bar{x}, c}, w_{i}^{\bar{x}}\right)$, the edge $\left(m^{\bar{x}, c}, w^{\bar{x}, c}\right)$ is the only balanced or man-heavy 
edge that is incident to man $m^{\bar{x}, c}$ and the only balanced (or woman-heavy) edge incident to woman $w^{\bar{x}, c}$. Hence, stability of the edge $\left(m^{\bar{x}, c}, w^{\bar{x}, c}\right)$ requires a weight of 1 assigned to it when the weight assigned to input edge $\left(m^{\bar{x}, c}, w_{i}^{\bar{x}}\right)$ is 0 . Then, the output edge $\left(m^{\bar{x}, c}, w_{j}^{c}\right)$, which is also incident to the node $m^{\bar{x}, c}$, must have a weight of 0 as well.

We now consider the case $\alpha \in(3 / 2,2)$. First consider a VC-connector corresponding to a positive literal $x$ and a clause $c$ containing it (Figure 6b). The edge $\left(m^{x, c}, w^{x, c}\right)$ is the only balanced or man-heavy edge that is incident to man $m^{x, c}$ and, besides the input edge $\left(m_{i}^{x}, w^{x, c}\right)$, the only balanced (or woman-heavy) edge incident to woman $w^{x, c}$. Hence, stability of edge $\left(m^{x, c}, w^{x, c}\right)$ requires a weight of 1 assigned to it when the weight assigned to edge $\left(m_{i}^{x}, w^{x, c}\right)$ is 0 . Then, the output edge $\left(m^{x, c}, w_{j}^{c}\right)$, which is also incident to node $m^{x, c}$, must have a weight of 0 as well.

Finally, consider a VC-connector corresponding to a negative literal $\bar{x}$ and a clause $c$ containing it (Figure 6 d). Observe that $\left(m_{1}^{\bar{x}, c}, w_{1}^{\bar{x}, c}\right)$ is the only balanced or woman-heavy edge that is incident to woman $w_{1}^{\bar{x}, c}$ and, besides the input edge $\left(m_{1}^{\bar{x}, c}, w_{i}^{\bar{x}}\right)$, the only balanced or man-heavy edge incident to man $m_{1}^{\bar{x}, c}$. Also, $\left(m_{2}^{\bar{x}, c}, w_{2}^{\bar{x}, c}\right)$ is the only balanced or man-heavy edge that is incident to man $m_{2}^{\bar{x}, c}$ and, besides edge $\left(m_{1}^{\bar{x}, c}, w_{2}^{\bar{x}, c}\right)$, the only balanced or woman-heavy edge to woman $w_{2}^{\bar{x}, c}$. Furthermore, $\left(m_{3}^{\bar{x}, c}, w_{3}^{\bar{x}, c}\right)$ is the only balanced or man-heavy edge that is incident to woman $w_{3}^{\bar{x}, c}$ and, besides edges $\left(m_{3}^{\bar{x}, c}, w_{1}^{\bar{x}, c}\right)$ and $\left(m_{3}^{\bar{x}, c}, w_{2}^{\bar{x}, c}\right)$, the only balanced or man-heavy edge incident to man $m_{3}^{\bar{x}, c}$.

Hence, stability of edge $\left(m_{1}^{\bar{x}, c}, w_{1}^{\bar{x}, c}\right)$ requires a weight of 1 assigned to it when the weight assigned to edge $\left(m_{1}^{\bar{x}, c}, w_{i}^{\bar{x}}\right)$ is 0 . Then, edges $\left(m_{1}^{\bar{x}, c}, w_{2}^{\bar{x}, c}\right)$ and $\left(m_{3}^{\bar{x}, c}, w_{1}^{\bar{x}, c}\right)$ must have a weight of 0 . Then, stability of edge $\left(m_{2}^{\bar{x}, c}, w_{2}^{\bar{x}, c}\right)$ requires a weight of 1 assigned to it and the edge $\left(m_{3}^{\bar{x}, c}, w_{2}^{\bar{x}, c}\right)$ and the output edge $\left(m_{2}^{\bar{x}, c}, w_{j}^{c}\right)$ must have a weight of 0 . Then, stability of edge $\left(m_{3}^{\bar{x}, c}, w_{3}^{\bar{x}, c}\right)$ requires a weight of 1 assigned to it. Hence, the output edge $\left(m_{3}^{\bar{x}, c}, w_{j}^{c}\right)$ must have a weight of 0 as well.

Claim 3. Any stable fractional matching that assigns a weight of 0 to all output edges of the VC-connectors of clause c must assign a weight of 0 to the CA-connector of clause $c$ as well.

Proof. Let $\ell_{1}, \ell_{2}$, and $\ell_{3}$ be the literals of clause $c$. Consider, for the sake of contradiction, a stable fractional matching that assigns (1) a weight of 0 to all output edges of the VC-connectors corresponding to literals $\ell_{i}$ and clause $c$, and (2) strictly positive weight to edge $\left(m^{c}, w^{c}\right)$ of the CA-connector for clause $c$. Note that condition (2) implies that the total weight on the edges $\left(m^{c}, w_{1}^{c}\right),\left(m^{c}, w_{2}^{c}\right)$ and $\left(m^{c}, w_{3}^{c}\right)$ is strictly smaller than 1. Since these are the only balanced or man-heavy edges incident to man $m^{c}$, the stability of these edges is guaranteed by a utility of (at least) 1 for each of the agents $w_{1}^{c}, w_{2}^{c}$, and $w_{3}^{c}$.

Note that besides the output edges of the VC-connectors, the edges $\left(e_{1}^{c}, w_{i}^{c}\right),\left(e_{2}^{c}, w_{i}^{c}\right)$, and $\left(m^{c}, w_{i}^{c}\right)$ are the only balanced or woman-heavy edges incident to agent $w_{i}^{c}$ for all $i \in\{1,2,3\}$. Along with condition (1), this implies that the weight assigned to these three edges is at least 1 . Hence, the total weight on the nine edges of the clause gadget is at least 3 , i.e., strictly more than 2 for the six edges incident to men $e_{1}^{c}$ and $e_{2}^{c}$, 
violating the definition of a fractional matching.

Claim 4. Any stable fractional matching that assigns a weight of 0 to some CA-connector must assign a total weight of 1 to the tine edges and a weight of 1 to every balanced edge of the accumulator.

615

Proof. Assume that a weight of 0 has been assigned to the edge $\left(m^{c^{\prime}}, w^{c^{\prime}}\right)$ of the CA-connector corresponding to some clause $c^{\prime}$. Since this is the only woman-heavy edge that is incident to agent $w^{c^{\prime}}$ and there is no man-heavy edge incident to agent $m_{1}$, stability on the edge $\left(m_{1}, w^{c^{\prime}}\right)$ requires that the total weight of the tine edges $\left(m_{1}, w^{c}\right)$ (for every clause $c$ ) is (at least) 1 . Hence, the weight of the edge $\left(m_{1}, w_{1}\right)$ is 0 . We will complete the proof by distinguishing between the two different accumulator structures, depending on whether $\alpha \geq 2$ or $\alpha \in(3 / 2,2)$.

When $\alpha \geq 2$, it suffices to show that for all $i \in\{1, \ldots, k-1\}$, if the weight of edge $\left(m_{i}, w_{i}\right)$ is 0 , then the weight of the balanced edges $\left(e_{i}^{1}, w_{i}\right)$ and $\left(m_{i+1}, f_{i+1}^{2}\right)$ is 1 and the weight of edge $\left(m_{i+1}, w_{i+1}\right)$ is 0 . Indeed, observe that, edge $\left(e_{i}^{1}, w_{i}\right)$ is the only balanced or man-heavy edge incident to man $e_{i}^{1}$ and, besides edge $\left(m_{i}, w_{i}\right)$, the only balanced or woman-heavy edge incident to woman $w_{i}$. Hence, the balanced edge $\left(e_{i}^{1}, w_{i}\right)$ must have a weight of 1 and the edge $\left(m_{i+1}, w_{i}\right)$ a weight of 0 .

Then, edge $\left(m_{i+1}, f_{i+1}^{2}\right)$ is the only balanced or woman-heavy edge incident to woman $f_{i+1}^{2}$ and, besides edge $\left(m_{i+1}, w_{i}\right)$, the only balanced or man-heavy edge incident to man $m_{i+1}$. Hence, the balanced edge $\left(m_{i+1}, f_{i+1}^{2}\right)$ must have a weight of 1 and the edge $\left(m_{i+1}, w_{i+1}\right)$ a weight of 0 .

When $\alpha \in(3 / 2,2)$, it suffices to show that for all $i \in\{1, \ldots, k-1\}$, if the weight of the edge $\left(m_{i}, w_{i}\right)$ (and, if they exist, the edges $\left(e_{i-1}^{1}, w_{i}\right)$ and $\left.\left(m_{i+1}, f_{i}^{2}\right)\right)$ is 0 , then the weight of the balanced edges $\left(e_{i}^{1}, w_{i}\right)$ and $\left(m_{i+1}, f_{i+1}^{2}\right)$ is 1 , and the weight of the edges $\left(m_{i+1}, w_{i+1}\right)$ and $\left(e_{i}^{1}, w_{i+1}\right)$ (and, if it exists, the edge $\left.\left(m_{i+2}, f_{i+1}^{2}\right)\right)$ is 0 . Indeed, observe that the edge $\left(e_{i}^{1}, w_{i}\right)$ is the only balanced or man-heavy edge incident to man $e_{i}^{1}$. Furthermore, in addition to the edge $\left(m_{i}, w_{i}\right)$ (and, if it exists, the edge $\left.\left(e_{i-1}^{1}, w_{i}\right)\right)$, it is the only balanced or woman-heavy edge incident to woman $w_{i}$. Hence, the balanced edge $\left(e_{i}^{1}, w_{i}\right)$ must have a weight of 1 , and the edge $\left(m_{i+1}, w_{i}\right)$ (and, if it exists, the edge $\left.\left(e_{i}^{1}, w_{i+1}\right)\right)$ must have a weight of 0 . Then, the edge $\left(m_{i+1}, f_{i+1}^{2}\right)$ is, besides $\left(m_{i+1}, f_{i}^{2}\right)$ and $\left(m_{i+1}, w_{i}\right)$, the only balanced or man-heavy edge incident to man $m_{i+1}$, and the only balanced or woman-heavy edge incident to woman $f_{i+1}^{2}$. Hence, the balanced edge $\left(m_{i+1}, f_{i+1}^{2}\right)$ must have a weight of 1 , and the edge $\left(m_{i+1}, w_{i+1}\right)\left(\right.$ and, if it exists, the edge $\left.\left(m_{i+2}, f_{i+1}^{2}\right)\right)$ must have a weight of 0 .

\subsection{Proof of inapproximability}

Lemma 4. If formula $\phi$ is not satisfiable, then any stable fractional matching of $\mathcal{I}$ has welfare at most $80 \alpha N+4(k-1)$.

Proof. We will first show that if $\phi$ is not satisfiable, then any stable fractional matching of $\mathcal{I}$ assigns weight 0 to some CA-connector. For the sake of contradiction, consider a stable fractional matching that assigns 
It can be easily seen that $80 \alpha N-2$ is a (loose) upper bound on the total value from these edges.

Lemma 5. If $\phi$ is satisfiable, then there exists a stable fractional matching of $\mathcal{I}$ with welfare at least $4(k-$ 1) $(\alpha-1 / 2)$.

Proof. Starting from a satisfying assignment for $\phi$, we will construct a stable fractional matching $\mu$ in which 675

a strictly positive weight to all CA-connectors. We will construct a truth assignment for the formula $\phi$ (contradicting the assumption of the lemma) by repeating the following process for every clause $c$ of $\phi$ : Let $\ell$ be a literal that appears in $c$ such that the output edge(s) of the VC-connector, that corresponds to the appearance of $\ell$ in $c$, have strictly positive total weight. By Claim 3 , such a literal must exist. We set $\ell$ to 1 (true). For every variable that has not received a value in this way, we arbitrarily set it to 1.

The above assignment satisfies all the clauses. To show that it is also valid, we need to argue that there is no variable $x$ such that both literals $x$ and $\bar{x}$ have been set to 1 . Assume, to the contrary, that literal $x$ is set to 1 due to its appearance in a clause $c_{1}$, and literal $\bar{x}$ is set to 1 due to its appearance in a different clause $c_{2}$. Thus, in the above assignment, the output edge(s) of the VC-connector between the literal $x$ and the clause $c_{1}$, as well as the VC-connector between the literal $\bar{x}$ and the clause $c_{2}$ have strictly positive (total) weight. By Claim 2, the input edges of both VC-connectors also have strictly positive weight. Let $i_{1}, i_{2} \in\{1,2\}$ be such that the $i_{1}$-th appearance of $x$ is in the clause $c_{1}$ and the $i_{2}$-th appearance of $\bar{x}$ is in the clause $c_{2}$. Therefore, the said input edges are incident to the nodes $m_{i_{1}}^{x}$ and $w_{i_{2}}^{\bar{x}}$. Using Claim 1 we get that the total weight on the edges incident to one of $m_{i_{1}}^{x}$ or $w_{i_{2}}^{\bar{x}}$ exceeds 1 , contradicting feasibility. Thus, the above assignment must be valid, which, in turn, implies that any stable fractional matching assigns weight 0 to some CA-connector.

By Claim 4 , the contribution of the accumulator to the welfare is exactly $4 k-2$ (2 from the tine edges plus 2 from each balanced edge). Let us now consider the contribution of the edges that do not belong to the accumulator. This comprises of

- a total value of 20 for the ten balanced edges of each of the $N$ variable gadgets,

- a total value of $\alpha$ (respectively, $2+2 \alpha$ ) for the edges of each of the $2 N \mathrm{VC}$-connectors corresponding to a positive literal when $\alpha \geq 2$ (respectively, $\alpha \in(3 / 2,2)$ ),

- a total value of $2+2 \alpha$ (respectively, $6+6 \alpha$ ) for the edges of each of the $2 N$ VC-connectors corresponding to a negative literal when $\alpha \geq 2$ (respectively, $\alpha \in(3 / 2,2)$ ),

- a total value of $18+\alpha$ for the nine balanced edges of each of the $4 N / 3$ clause gadgets and their corresponding CA-connectors.

the welfare of the accumulator gadget is at least $4(k-1)(\alpha-1 / 2)$. 
Variable gadgets.. For the edges of the variable gadget of the variable $x, \mu$ is defined as:

- If $x$ is true, then $\mu\left(m_{1}^{x}, w_{1}^{\bar{x}}\right)=\mu\left(e_{3}^{x}, w_{1}^{\bar{x}}\right)=\mu\left(e_{3}^{x}, w_{2}^{\bar{x}}\right)=\mu\left(m_{2}^{x}, w_{2}^{\bar{x}}\right)=1 / 2, \mu\left(e_{1}^{x}, f_{1}^{x}\right)=\mu\left(e_{2}^{x}, f_{2}^{x}\right)=1$, and the remaining edges have weight 0 .

- If $x$ is false, then $\mu\left(e_{3}^{x}, w_{1}^{\bar{x}}\right)=\mu\left(e_{3}^{x}, w_{2}^{\bar{x}}\right)=1 / 2, \mu\left(m_{1}^{x}, f_{1}^{x}\right)=\mu\left(m_{2}^{x}, f_{2}^{x}\right)=1$, and the remaining edges have weight 0.

Clause gadgets and CA-connectors. For each clause, select one of the true literals (tie-break arbitrarily) and call it active. Note that each clause has an active literal in a satisfying assignment. Consider the clause $c$, and let $\ell_{i}$ be its active literal for some $i \in\{1,2,3\}$. Also, let $i_{1}, i_{2} \in\{1,2,3\} \backslash\{i\}$ denote the other two indices. Set $\mu\left(e_{1}^{c}, w_{i_{1}}^{c}\right)=\mu\left(e_{2}^{c}, w_{i_{2}}^{c}\right)=1$, and set the weight of the remaining balanced edges to 0 . Assign a weight of 1 to the CA-connector, i.e., $\mu\left(m^{c}, w^{c}\right)=1$.

$V C$-connectors.. For every non-active VC-connector, set the weight of its balanced edges (if any) to 1 and the weight of the remaining edges to 0 . For every active VC-connector corresponding to the $i$-th appearance of the positive literal $x$ as the $j$-th literal of clause $c(i \in\{1,2\}, j \in\{1,2,3\})$, the weights of its edges are as follows:

- When $\alpha \geq 2$, we set $\mu\left(m_{i}^{x}, w_{j}^{c}\right)=1 / 2$.

- When $\alpha \in(3 / 2,2)$, we set $\mu\left(m_{i}^{x}, w^{x, c}\right)=1 / 2, \mu\left(m^{x, c}, w^{x, c}\right)=1-\alpha / 2$, and $\mu\left(m^{x, c}, w_{j}^{c}\right)=1 / \alpha$.

For every active VC-connector corresponding to the $i$-th appearance of the negative literal $x$ as the $j$-th literal of clause $c(i \in\{1,2\}, j \in\{1,2,3\})$, the weights of its edges are as follows:

- When $\alpha \geq 2$, we set $\mu\left(m^{\bar{x}, c}, w_{i}^{\bar{x}}\right)=\mu\left(m^{\bar{x}, c}, w_{j}^{c}\right)=1 / 2$ and $\mu\left(m^{\bar{x}, c}, w^{\bar{x}, c}\right)=0$.

- When $\alpha \in(3 / 2,2)$, we set $\mu\left(m_{1}^{\bar{x}, c}, w_{i}^{\bar{x}}\right)=1 / 2, \mu\left(m_{1}^{\bar{x}, c}, w_{1}^{\bar{x}, c}\right)=1-\alpha / 2, \mu\left(m_{1}^{\bar{x}, c}, w_{2}^{\bar{x}, c}\right)=(\alpha-$ $1) / 2, \mu\left(m_{2}^{\bar{x}, c}, w_{2}^{\bar{x}, c}\right)=1-\left(\alpha^{2}-\alpha\right) / 2, \mu\left(m_{2}^{\bar{x}, c}, w_{j}^{c}\right)=2 / \alpha-1, \mu\left(m_{3}^{\bar{x}, c}, w_{1}^{\bar{x}, c}\right)=1 / \alpha, \mu\left(m_{3}^{\bar{x}, c}, w_{2}^{\bar{x}, c}\right)=$ $\mu\left(m_{3}^{\bar{x}, c}, w_{3}^{\bar{x}, c}\right)=0, \mu\left(m_{3}^{\bar{x}, c}, w_{j}^{c}\right)=1-1 / \alpha$.

Accumulator.. We set $\mu\left(m_{1}, w^{c}\right)=0$ for every tine edge $\left(m_{1}, w^{c}\right)$ of the accumulator. Furthermore:

- When $\alpha \geq 2$, we set $\mu\left(m_{i}, w_{i}\right)=1 / \alpha$ for all $i \in\{1, \ldots, k\}, \mu\left(e_{i}^{2}, w_{i}\right)=1-2 / \alpha, \mu\left(m_{i+1}, w_{i}\right)=1 / \alpha$, $\mu\left(e_{i}^{1}, f_{i}^{1}\right)=1, \mu\left(e_{i}^{1}, w_{i}\right)=0$ for all $i \in\{1, \ldots, k-1\}, \mu\left(m_{i}, f_{i}^{2}\right)=0, \mu\left(m_{i}, f_{i}^{3}\right)=1-2 / \alpha$, and $\mu\left(e_{i}^{3}, f_{i}^{2}\right)=1$ for all $i \in\{2, \ldots, k\}$. Among these, any edge with a positive weight is either man- or woman-heavy, and hence, its contribution to the social welfare is $\alpha$ times its weight. It can be verified that the total contribution is $4(k-1)(\alpha-1 / 2)+1$. 
- When $\alpha \in(3 / 2,2)$, we set $\mu\left(m_{1}, w_{1}\right)=1 / \alpha, \mu\left(m_{2}, w_{2}\right)=\alpha+1 / \alpha-2, \mu\left(m_{i}, w_{i}\right)=1-1 / \alpha$ for all $i \in\{3, \ldots, k\}, \mu\left(m_{i+1}, w_{i}\right)=1-1 / \alpha$ for all $i \in\{1, \ldots, k-1\}, \mu\left(e_{i}^{1}, w_{i}\right)=0$ for all $i \in\{1, \ldots, k-1\}$, $\mu\left(m_{2}, f_{2}^{2}\right)=2-\alpha, \mu\left(m_{i}, f_{i}^{2}\right)=0$ for all $i \in\{3, \ldots, k\}, \mu\left(e_{1}^{1}, f_{1}^{1}\right)=\alpha-1, \mu\left(e_{2}^{2}, f_{2}^{2}\right)=\alpha-2 / \alpha$, $\mu\left(e_{k}^{2}, f_{k}^{2}\right)=1, \mu\left(e_{i}^{1}, f_{i}^{1}\right)=2-2 / \alpha$ for all $i \in\{2, \ldots, k-1\}, \mu\left(e_{i}^{2}, f_{i}^{2}\right)=2-2 / \alpha$ for all $i \in\{3, \ldots, k-1\}$, $\mu\left(e_{1}^{1}, w_{2}\right)=2-\alpha, \mu\left(e_{i}^{1}, w_{i+1}\right)=2 / \alpha-1$ for all $i \in\{2, \ldots, k-1\}$, and $\mu\left(m_{i+1}, f_{i}^{2}\right)=2 / \alpha-1$ for all $i \in$ $\{2, \ldots, k-1\}$. Except for the balanced edge $\left(m_{2}, f_{2}^{2}\right)$, every edge with a positive weight among the ones listed above is either man- or woman-heavy, and hence, its contribution to the social welfare is $\alpha$ times its weight. It can be verified that the total contribution in this case is $4(k-1)(\alpha-1 / 2)+2 \alpha^{2}-7 \alpha+7$.

In each case, the accumulator contributes at least $4(k-1)(\alpha-1 / 2)$ to the social welfare, as desired.

The feasibility of $\mu$ can be verified by inspection. To see why $\mu$ is stable, note that we only need to check for the balanced edges, as the man- or woman-heavy edges and the remaining pairs do not impose any constraints on stability. For the balanced edges, stability is established by the following series of observations (we will use the term 'stabilized by' to denote that an agent's utility is at least 1): The variable gadget for the variable $x$ (Figure 5 ) is stabilized by the agents $f_{1}^{x}, f_{2}^{x}, e_{3}^{x}$ along with $m_{1}^{x}, m_{2}^{x}$ (if $x$ is true) or $w_{1}^{\bar{x}}, w_{2}^{\bar{x}}$ (if $x$ is false). The clause gadget for clause $c$ (Figure 5b) with active index $i$ (and non-active indices $i_{1}$ and $\left.i_{2}\right)$ is stabilized by the agents $e_{1}^{c}, e_{2}^{c}, w_{i}^{c}, w_{i_{1}}^{c}, w_{i_{2}}^{c}$; in particular, the edge $\left(m^{c}, w_{i}^{c}\right)$ is stabilized by $w_{i}^{c}$ because an active literal triggers the woman-heavy edge in the VC-connector. A $\mathrm{VC}$ connector is stabilized by $w^{x, c}$ (Figure 6b), $m^{\bar{x}, c}$ (Figure 6c), or $m_{1}^{\bar{x}, c}, w_{2}^{\bar{x}, c}$, and $m_{3}^{\bar{x}, c}$ (Figure 6d). Finally, the tine edges in the accumulator (Figure 7) are stabilized by $w^{c_{1}}, \ldots, w^{c_{L}}$ (because we trigger the CA-connector), and the remaining balanced edges are stabilized by $w_{i}$ 's and $m_{i}$ 's except for $m_{1}$. Overall, $\mu$ is a feasible stable fractional matching.

We are ready to prove Theorem 7 . If $\alpha<N^{1+1 / \delta}$, we use our construction with any $k$ satisfying $k-1 \geq \frac{20 \alpha N(\alpha-1 / 2-\delta)}{\delta}$. It is easy to verify that the reduction is polynomial-time. Furthermore, from Lemma 4, we know that the welfare of $\mu$ when $\phi$ is not satisfiable is at most

$$
80 \alpha N+4(k-1) \leq \frac{4(k-1) \delta}{\alpha-1 / 2-\delta}+4(k-1)=\frac{4(k-1)(\alpha-1 / 2)}{\alpha-1 / 2-\delta} .
$$

This number is at least $\alpha-1 / 2-\delta$ times smaller than the welfare of $\mu$ when $\phi$ is satisfiable (Lemma 5). This establishes the inapproximability bound in part (i) of Theorem 7 .

If $\alpha \geq N^{1+1 / \delta}$, we use our construction with $k=N^{1+1 / \delta}$. Once again, the reduction is polynomial-time, and the instance $\mathcal{I}$ has $n=\Theta\left(N^{1+1 / \delta}\right)$ men and women. Observe that $\alpha=\Omega(n), k=\Theta(n)$, and $N=\mathcal{O}\left(n^{\delta}\right)$. Hence, the welfare of $\mu$ when $\phi$ is not satisfiable is at most

$$
80 \alpha N+4(k-1) \leq 80 \alpha N+4 N^{1+1 / \delta} \leq 84 \alpha N=\mathcal{O}\left(\alpha n^{\delta}\right) .
$$

On the other hand, the welfare of $\mu$ when $\phi$ is satisfiable is at least $4(k-1)(\alpha-1 / 2)$, i.e., $\Omega(\alpha n)$. This establishes the bound in part (ii), and with it, completes the proof of Theorem 7 


\section{Concluding Remarks}

We studied stable fractional matchings in a cardinal model and provided a number of computational

and structural results. Going forward, it would be very interesting to resolve the complexity of OpTIMAL Stable Fractional Matching for the case when agents have strict cardinal preferences (i.e., the no-ties case). It would also be very interesting to see if stronger inapproximability results can be obtained for more general matching models, such as stable roommates [59. Another relevant direction is to consider a stronger solution concept with blocking coalitions of any size (also known as the core), wherein the deviating agents can form a fractional matching among themselves to achieve a higher utility for each member of the coalition.

\section{Acknowledgments.}

We are grateful to Elliot Anshelevich for bringing the work of Deligkas et al. 47] to our attention, to Argyrios Deligkas for sharing with us the full version of their paper [4], and to Haris Aziz for pointing us to the work of Manjunath [48. Many thanks to the anonymous reviewers for their thoughtful comments and suggestions. Ioannis Caragiannis and Aris Filos-Ratsikas acknowledge partial support from COST Action CA15210. Rohit Vaish acknowledges support from ONR\#N00014-17-1-2621 while he was affiliated with Rensselaer Polytechnic Institute, and is currently supported by project no. RTI4001 of the Department of Atomic Energy, Government of India. Part of this work was done while Rohit Vaish was supported by the Prof. R Narasimhan postdoctoral award.

\section{References}

[1] D. Gale, L. S. Shapley, College Admissions and the Stability of Marriage, The American Mathematical Monthly 69 (1) (1962) 9-15.

[2] D. Gusfield, R. W. Irving, The Stable Marriage Problem: Structure and Algorithms, MIT Press, 1989.

[3] A. E. Roth, M. A. O. Sotomayor, Two-Sided Matching: A Study in Game-Theoretic Modeling and Analysis, Cambridge University Press, 1990.

[4] D. E. Knuth, Stable Marriage and its Relation to Other Combinatorial Problems: An Introduction to the Mathematical Analysis of Algorithms, Vol. 10, American Mathematical Soc., 1997.

[5] D. F. Manlove, Algorithmics of Matching under Preferences, Vol. 2, World Scientific, 2013.

[6] A. E. Roth, The Evolution of the Labor Market for Medical Interns and Residents: A Case Study in Game Theory, Journal of Political Economy 92 (6) (1984) 991-1016. 
[7] A. E. Roth, E. Peranson, The Redesign of the Matching Market for American Physicians: Some Engineering Aspects of Economic Design, American Economic Review 89 (4) (1999) 748-780.

[8] A. Abdulkadiroğlu, P. A. Pathak, A. E. Roth, The New York City High School Match, American Economic Review (2005) 364-367.

[19] E. Anshelevich, S. Das, Y. Naamad, Anarchy, Stability, and Utopia: Creating Better Matchings, Autonomous Agents and Multi-Agent Systems 26 (1) (2013) 120-140.

[20] D. Gusfield, Three Fast Algorithms for Four Problems in Stable Marriage, SIAM Journal on Computing 16 (1) (1987) 111-128. 
[21] R. W. Irving, P. Leather, D. Gusfield, An Efficient Algorithm for the "Optimal" Stable Marriage, Journal of the ACM 34 (3) (1987) 532-543.

[22] C.-P. Teo, J. Sethuraman, The Geometry of Fractional Stable Matchings and its Applications, Mathematics of Operations Research 23 (4) (1998) 874-891.

[23] D. F. Manlove, R. W. Irving, K. Iwama, S. Miyazaki, Y. Morita, Hard Variants of Stable Marriage, Theoretical Computer Science 276 (1-2) (2002) 261-279.

[24] T. Mai, V. V. Vazirani, A Natural Generalization of Stable Matching Solved via New Insights into Ideal Cuts, arXiv preprint arXiv:1802.06621.

[25] A. Kato, Complexity of the Sex-Equal Stable Marriage Problem, Japan Journal of Industrial and Applied Mathematics 10 (1) (1993) 1.

[26] T. Feder, A New Fixed Point Approach for Stable Networks and Stable Marriages, Journal of Computer and System Sciences 45 (2) (1992) 233-284.

[27] K. Iwama, S. Miyazaki, Y. Morita, D. F. Manlove, Stable Marriage with Incomplete Lists and Ties, in: International Colloquium on Automata, Languages, and Programming, Springer, 1999, pp. 443-452.

[28] R. W. Irving, D. F. Manlove, G. O'Malley, Stable Marriage with Ties and Bounded Length Preference Lists, Journal of Discrete Algorithms 7 (2) (2009) 213-219.

[29] J. Drummond, C. Boutilier, Elicitation and Approximately Stable Matching with Partial Preferences, in: Twenty-Third International Joint Conference on Artificial Intelligence, 2013, pp. 97-105.

[30] B. Rastegari, P. Goldberg, D. F. Manlove, Preference Elicitation in Matching Markets via Interviews: A Study of Offline Benchmarks, in: Proceedings of the 2016 International Conference on Autonomous Agents \& Multiagent Systems, 2016, pp. 1393-1394.

[31] M. Goto, A. Iwasaki, Y. Kawasaki, R. Kurata, Y. Yasuda, M. Yokoo, Strategyproof Matching with Regional Minimum and Maximum Quotas, Artificial Intelligence 235 (2016) 40-57.

[32] N. Hamada, C.-L. Hsu, R. Kurata, T. Suzuki, S. Ueda, M. Yokoo, Strategy-Proof School Choice Mechanisms with Minimum Quotas and Initial Endowments, Artificial Intelligence 249 (2017) 47-71.

[33] I. P. Gent, P. Prosser, B. Smith, T. Walsh, SAT Encodings of the Stable Marriage Problem with Ties and Incomplete Lists, in: Fifth International Symposium on the Theory and Applications of Satisfiability Testing, 2002, pp. 133-140. 
[34] J. Drummond, A. Perrault, F. Bacchus, SAT is an Effective and Complete Method for Solving Stable Matching Problems with Couples, in: Twenty-Fourth International Joint Conference on Artificial Intelligence, 2015 , pp. 518-525.

[35] A. Perrault, J. Drummond, F. Bacchus, Strategy-Proofness in the Stable Matching Problem with Couples, in: Proceedings of the 2016 International Conference on Autonomous Agents and Multiagent Systems, 2016, pp. 132-140.

[36] I. P. Gent, R. W. Irving, D. F. Manlove, P. Prosser, B. M. Smith, A Constraint Programming Approach to the Stable Marriage Problem, in: International Conference on Principles and Practice of Constraint Programming, Springer, 2001, pp. 225-239.

[37] D. F. Manlove, G. OMalley, P. Prosser, C. Unsworth, A Constraint Programming Approach to the Hospitals/Residents Poblem, in: International Conference on Integration of Artificial Intelligence (AI) and Operations Research (OR) Techniques in Constraint Programming, Springer, 2007, pp. 155-170.

[38] D. F. Manlove, I. McBride, J. Trimble, "Almost-stable" matchings in the Hospitals/Residents problem with Couples, Constraints 22 (1) (2017) 50-72.

[39] U. Endriss, Analysis of One-to-One Matching Mechanisms via SAT Solving: Impossibilities for Universal Axioms, in: Proceedings of the Thirty-Fourth AAAI Conference on Artificial Intelligence (forthcoming), AAAI Press, 2020.

[40] J. H. Vande Vate, Linear Programming Brings Marital Bliss, Operations Research Letters 8 (3) (1989) $147-153$.

[41] U. G. Rothblum, Characterization of Stable Matchings as Extreme Points of a Polytope, Mathematical Programming 54 (1-3) (1992) 57-67.

[42] R. V. Vohra, Stable Matchings and Linear Programming, Current Science 105 (9) (2012) 1051-1055.

[43] X. Chen, G. Ding, X. Hu, W. Zang, The Maximum-Weight Stable Matching Problem: Duality and Efficiency, SIAM Journal on Discrete Mathematics 26 (3) (2012) 1346-1360.

[44] R. Aharoni, T. Fleiner, On a Lemma of Scarf, Journal of Combinatorial Theory, Series B 87 (1) (2003) $72-80$.

[45] P. Biró, T. Fleiner, Fractional Solutions for Capacitated NTU-Games, with Applications to Stable Matchings, Discrete Optimization 22 (2016) 241-254.

[46] H. Aziz, B. Klaus, Random Matching under Priorities: Stability and No Envy Concepts, Social Choice and Welfare (2019) 1-47. 
[47] A. Deligkas, G. B. Mertzios, P. G. Spirakis, The Computational Complexity of Weighted Greedy Matching, in: Proceedings of the Thirty-First AAAI Conference on Artificial Intelligence, AAAI Press, 2017,

[48] V. Manjunath, Stability and the Core of Probabilistic Marriage Problems, Available at SSRN 1809941.

[49] J. W. Hatfield, S. D. Kominers, A. Nichifor, M. Ostrovsky, A. Westkamp, Stability and Competitive Equilibrium in Trading Networks, Journal of Political Economy 121 (5) (2013) 966-1005.

[50] J. W. Hatfield, S. D. Kominers, Multilateral Matching, Journal of Economic Theory 156 (2015) 175-206.

[51] B. Doğan, K. Yıldız, Efficiency and Stability of Probabilistic Assignments in Marriage Problems, Games and Economic Behavior 95 (2016) 47-58.

[52] F. Echenique, A. Galichon, Ordinal and Cardinal Solution Concepts for Two-Sided Matching, Games and Economic Behavior 101 (2017) 63-77.

[53] K. C. Ágoston, P. Biró, I. McBride, Integer Programming Methods for Special College Admissions Problems, Journal of Combinatorial Optimization 32 (4) (2016) 1371-1399.

[54] M. Delorme, S. García, J. Gondzio, J. Kalcsics, D. Manlove, W. Pettersson, Mathematical Models for Stable Matching Problems with Ties and Incomplete Lists, European Journal of Operational Research 277 (2) (2019) 426-441.

[55] S. Narang, Y. Narahari, A Study of Incentive Compatibility and Stability Issues in Fractional Matchings, arXiv preprint arXiv:2001.05652.

[56] R. J. Lipton, E. Markakis, A. Mehta, Playing Large Games using Simple Strategies, in: Proceedings of the Fourth ACM Conference on Electronic Commerce, ACM, 2003, pp. 36-41.

[57] S. Barman, Approximating Nash Equilibria and Dense Subgraphs via an Approximate Version of Carathéodory's Theorem, SIAM Journal on Computing 47 (3) (2018) 960-981.

[58] R. Yoshinaka, Higher-Order Matching in the Linear Lambda Calculus in the Absence of Constants is NP-Complete, in: Proceedings of the Sixteenth International Conference on Term Rewriting and Applications, Springer-Verlag, 2005, pp. 235-249.

[59] R. W. Irving, An Efficient Algorithm for the "Stable Roommates" Problem, Journal of Algorithms 6 (4) (1985) 577-595. 


\section{Comparison with other notions of stability}

In this section, we will discuss other notions of stability of fractional matchings that have been studied for ordinal preferences. For a detailed overview of these and other notions, we refer the reader to the work of Aziz and Klaus [46].

Given an SMC instance $\mathcal{I}=\langle M, W, U, V\rangle$ with cardinal preferences, we can define an ordinal instance $\mathcal{I}^{\text {ord }}=\langle M, W, \succeq\rangle$ where $\succeq:=\left(\succeq_{m_{1}}, \ldots, \succeq_{m_{n}}, \succeq_{w_{1}}, \ldots, \succeq_{w_{n}}\right)$ is a preference profile consisting of the ordinal preferences of the agents specified as weak total orders. Specifically, for every $i \in\{1, \ldots, n\}, \succeq_{m_{i}}$ and $\succeq_{w_{i}}$ are weak total orders over the sets $W$ and $M$, respectively, such that $U\left(m_{i}, w\right) \geq U\left(m_{i}, w^{\prime}\right) \Leftrightarrow w \succeq_{m_{i}} w^{\prime}$ and $V\left(m, w_{i}\right) \geq V\left(m^{\prime}, w_{i}\right) \Leftrightarrow m \succeq_{w_{i}} m^{\prime}$. We will write $w \succ_{m} w^{\prime}$ if $w \succeq_{m} w^{\prime}$ but not $w^{\prime} \succeq_{m} w$. The relation $m \succ_{w} m^{\prime}$ is analogously defined.

Below we will discuss three notions of stability for fractional matchings - strong stability [11, fractional stability [40], and ex-post stability [11] - that were originally formulated in the context of strict ordinal preferences, and were subsequently studied for weak preferences by Aziz and Klaus [46].

The first notion called strong stability asserts that no pair of man and woman should positively engage with agents they like less than each other.

Definition 5 (Strong stability [11]). A fractional matching $\mu$ is strongly stable if there are no $m, m^{\prime} \in M$ and $w, w^{\prime} \in W$ such that $\mu\left(m, w^{\prime}\right)>0, \mu\left(m^{\prime}, w\right)>0, w \succ_{m} w^{\prime}$, and $m \succ_{w} m^{\prime}$.

Next, recall that a fractional matching can be interpreted as a lottery (or a probability distribution) over integral matchings. Under this interpretation, ex-post stability requires that every realization of this lottery should be stable.

Definition 6 (Ex-post stability [11]). A fractional matching $\mu$ is ex-post stable if it can be expressed as a convex combination of integral stable matchings.

The third notion, fractional stability, formalizes the idea that "if man $m$ is matched with someone less preferred than woman $w$, then $w$ should be matched with someone more preferred than $m$ " as a linear constraint.

Definition 7 (Fractional stability [40]). A fractional matching $\mu$ is fractionally stable if for every $(m, w) \in$ $M \times W$,

$$
\mu(m, w)+\sum_{w^{\prime} \in W: w^{\prime} \succeq_{m} w} \mu\left(m, w^{\prime}\right)+\sum_{m^{\prime} \in M: m^{\prime} \succeq_{w} m} \mu\left(m^{\prime}, w\right) \geq 1 .
$$

Notice that an integral matching satisfies the ordinal notions of strong (Definition 5), ex-post (Definition 6), or fractional (Definition 7) stability in $\mathcal{I}^{\text {ord }}$ if and only if it satisfies the cardinal notion of stability (Definition 1) in $\mathcal{I}$. Thus, the distinction between these notions is meaningful only for fractional matchings. The following observations describe the relationship between these notions (also refer to Figure 8). 


\section{Ordinal Preferences}

Cardinal Preferences

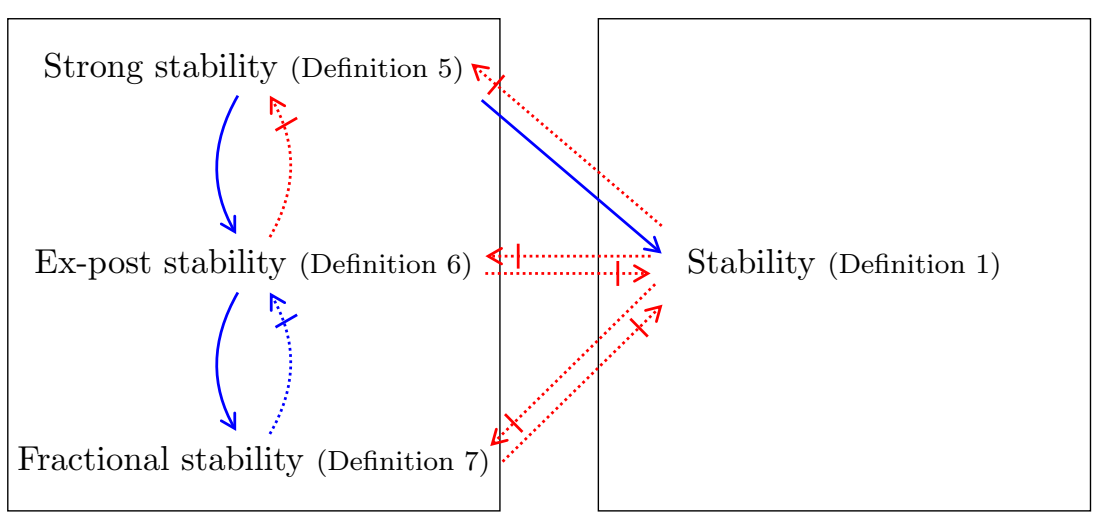

Figure 8: Relationship between stability notions for fractional matchings under ordinal and cardinal preferences. A solid (respectively, dotted) arrow indicates that the implication holds (respectively, does not hold) in that direction. The implications for strict and weak preferences are denoted by red and blue arrows, respectively.

- It is known that strong stability implies ex-post stability even under weak ordinal preferences, but the converse is not true even under strict ordinal preferences [11,46]. Additionally, for strict preferences, expost stability is known to be equivalent to fractional stability [40, 41, 11, 22, but for weak preferences, the former is strictly stronger [46].

- A stable matching (Definition 1) may not be fractionally stable (Definition 7) even under strict preferences. To see this, consider the fractional matching $\mu$ in Example 1, which was shown to be stable. For the pair $\left(m_{1}, w_{3}\right)$, we have

$$
\mu\left(m_{1}, w_{3}\right)+\sum_{w^{\prime}: w^{\prime} \succ m_{1} w_{3}} \mu\left(m_{1}, w^{\prime}\right)+\sum_{m^{\prime}: m^{\prime} \succ w_{3} \succ m_{1}} \mu\left(m^{\prime}, w_{3}\right)=0+0+0.5<1,
$$

implying that $\mu$ is not fractionally stable (and thus also not ex-post/strongly stable).

Remark 3. It is interesting to note the welfare implication of the above observation. Since fractionally stable matchings are equivalent to ex-post stable matchings under strict preferences, and since all integral stable matchings in Example 1 have social welfare of 7, it follows that the social welfare of any fractionally stable matching is also equal to 7 . On the other hand, there exists a stable matching $\mu$ with a strictly higher welfare. This illustrates that fractionally stable (and, in particular, strongly stable) matchings can be strictly suboptimal in the cardinal model, further justifying the need to study the computational aspects of optimal stable matchings.

- Given an SMC instance $\mathcal{I}$, a strongly stable (Definition 5 matching in the corresponding ordinal instance $\mathcal{I}^{\text {ord }}$ can be shown to be stable (Definition 1) in the original instance $\mathcal{I}$. Indeed, if $\mu$ is strongly 
stable in $\mathcal{I}^{\text {ord }}$, then for any pair $(m, w) \in M \times W$, we have that either $\sum_{w^{\prime}: w \succ_{m} w^{\prime}} \mu\left(m, w^{\prime}\right)=0$ or $\sum_{m^{\prime}: m \succ w} m^{\prime}\left(m^{\prime}, w\right)=0$. Suppose, without loss of generality, that the former holds. Then, the utility of man $m$ in $\mathcal{I}$ is given by

$$
\begin{aligned}
u_{m}(\mu) & =U(m, w) \mu(m, w)+\sum_{w^{\prime}: w^{\prime} \succeq_{m} w} U\left(m, w^{\prime}\right) \mu\left(m, w^{\prime}\right) \\
& \geq U(m, w)\left(\mu(m, w)+\sum_{w^{\prime}: w^{\prime} \succ_{m} w} \mu\left(m, w^{\prime}\right)\right) \\
& =U(m, w),
\end{aligned}
$$

as desired.

- As mentioned previously in Remark 1, Narang and Narahari [55] have observed that the set of stable matchings is non-convex even under strict cardinal preferences. Their counterexample involves the convex combination of integral stable matchings, and therefore also establishes that an ex-post stable (and hence also fractionally stable) matching may not be stable.

\section{Non-convexity of strong stability may not imply non-convexity of stability}

In this section, we show that non-convexity of strong stability (Definition 5 might not imply the same for stability (Definition 1), even though, as observed in Section 7, the former is a strictly stronger notion. To this end, we revisit the counterexample used by Aziz and Klaus [46 in establishing the non-convexity of the set of strongly stable matchings. Recall from Section 7 that a fractional matching $\mu$ is strongly stable if there are no $m, m^{\prime} \in M$ and $w, w^{\prime} \in W$ such that $\mu\left(m, w^{\prime}\right)>0, \mu\left(m^{\prime}, w\right)>0, w \succ_{m} w^{\prime}$, and $m \succ_{w} m^{\prime}$.

The counterexample of Aziz and Klaus [46, Theorem 1], which in turn is adapted from [11, Example 2], consists of three men $m_{1}, m_{2}, m_{3}$ and three women $w_{1}, w_{2}, w_{3}$ with the following ordinal preferences:

$$
\begin{array}{ll}
m_{1}: w_{1} \succ w_{2} \succ w_{3} & w_{1}: m_{2} \succ m_{3} \succ m_{1} \\
m_{2}: w_{2} \succ w_{3} \succ w_{1} & w_{2}: m_{3} \succ m_{1} \succ m_{2} \\
m_{3}: w_{3} \succ w_{1} \succ w_{2} & w_{3}: m_{1} \succ m_{2} \succ m_{3} .
\end{array}
$$

Consider the integral matchings $\mu^{(1)}, \mu^{(2)}$, and $\mu^{(3)}$ defined as follows:

$$
\begin{aligned}
& \mu^{(1)}:=\left\{\left(m_{1}, w_{1}\right),\left(m_{2}, w_{2}\right),\left(m_{3}, w_{3}\right)\right\}, \\
& \mu^{(2)}:=\left\{\left(m_{1}, w_{3}\right),\left(m_{2}, w_{1}\right),\left(m_{3}, w_{2}\right)\right\}, \text { and } \\
& \mu^{(3)}:=\left\{\left(m_{1}, w_{2}\right),\left(m_{2}, w_{3}\right),\left(m_{3}, w_{1}\right)\right\} .
\end{aligned}
$$

Notice that $\mu^{(1)}, \mu^{(2)}$, and $\mu^{(3)}$ are strongly stable (and therefore also stable). Also note that the fractional matching $\mu:=\frac{1}{3} \mu^{(1)}+\frac{1}{3} \mu^{(2)}+\frac{1}{3} \mu^{(3)}$ violates strong stability for the agents $m_{1}, m_{2}$ and $w_{2}, w_{3}$ since 
$\mu\left(m_{1}, w_{3}\right)>0, \mu\left(m_{2}, w_{2}\right)>0, w_{2} \succ_{m_{1}} w_{3}$ and $m_{1} \succ_{w_{2}} m_{2}$. That is, the convex combination of strongly stable integral matchings $\mu^{(1)}, \mu^{(2)}$, and $\mu^{(3)}$ is not strongly stable.

Let us now consider an SMC instance $\mathcal{I}=\langle M, W, U, V\rangle$ for the same set of agents, i.e., $M=\left\{m_{1}, m_{2}, m_{3}\right\}$, $W=\left\{w_{1}, w_{2}, w_{3}\right\}$, and the following valuations:

$$
U=\left[\begin{array}{lll}
2 & 1 & 0 \\
0 & 2 & 1 \\
1 & 0 & 2
\end{array}\right] \text { and } V=\left[\begin{array}{ccc}
0 & 1 & 2 \\
2 & 0 & 1 \\
1 & 2 & 0
\end{array}\right]
$$

Observe that the valuations in $\mathcal{I}$ are consistent with the aforementioned ordinal preferences. It is easy to see that the utility of each agent in $\mu$ is equal to 1 , and that $\mu$ is stable for $\mathcal{I}$.

\section{Hardness for $\varepsilon$-stability}

The proof of Theorem 8 follows along very similar lines to the proof of Theorem 7 , again using a reduction from 2P2N-3SAT.

\subsection{The reduction}

Starting from an instance of $2 \mathrm{P} 2 \mathrm{~N}-3 \mathrm{SAT}$, we first preprocess and augment it in the following way. For each variable of the original instance, we create a copy-variable and, for each clause of the original instance, we create a copy-clause that contains the copy-variables corresponding to the variables of the original clause. Each variable and its copy are coupled variables and, similarly, each clause and its copy are coupled clauses.

Let the modified input consist of $N$ (boolean) variables $x_{1}, x_{2}, \ldots, x_{N}$, and a 3 -CNF formula $\phi$ with $L=4 N / 3$ clauses $c_{1}, c_{2}, \ldots, c_{4 N / 3}$. Note that if $\phi$ is not satisfiable, then, due to the instance augmentation, there exist at least two clauses that are not satisfied.

We now proceed to describe the instance $\mathcal{I}=\langle M, W, U, V\rangle$ of Optimal $\varepsilon$-Stable Fractional MatchING whose graph representation consists of variable gadgets, clause gadgets, VC-connectors, an accumulator, and CA-connectors. We remark that $\mathcal{I}$ is not an instance with ternary valuations as we use valuations from the set $\{0,1, \beta, \gamma\}$. We set $\beta=2(1-\varepsilon)$, and observe that, when $\varepsilon<0.03$, we have that $\left(3 \beta^{2}+4\right) \varepsilon<1 / 2$; the value of $\gamma$ will be set later. Again, we denote by $n$ the number of men (or women) in $\mathcal{I}$; the following reduction is such that $n=\mathcal{O}(N)$.

For each gadget, we classify the edges into the following three types:

- balanced edges $(m, w)$ with $U(m, w)=V(m, w)=1$,

- man-heavy edges $(m, w)$ with $U(m, w)>0$ and $V(m, w)=0$, and

- woman-heavy edges $(m, w)$ with $U(m, w)=0$ and $V(m, w)>0$. 


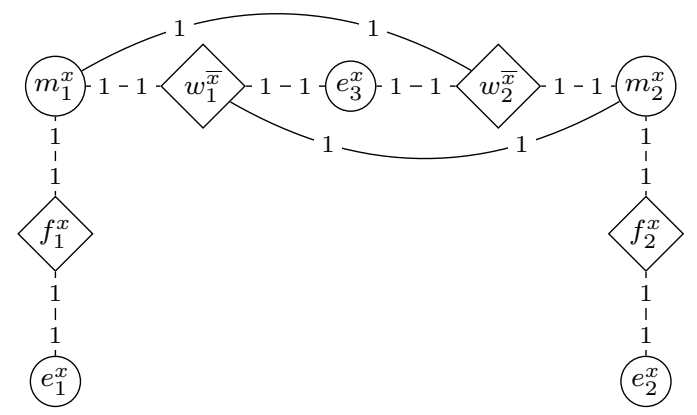

(a)

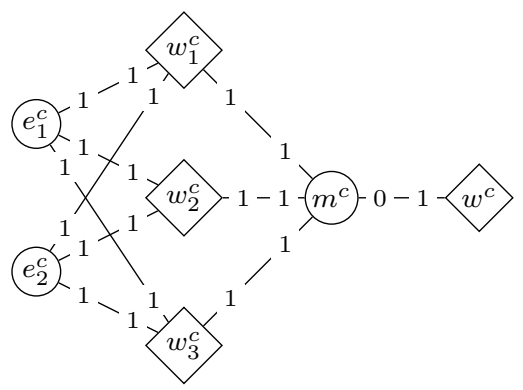

(b)

Figure 9: (a) The variable gadget corresponding to the variable $x$. (b) The clause gadget corresponding to the clause $c$ and its CA-connector $\left(m^{c}, w^{c}\right)$.

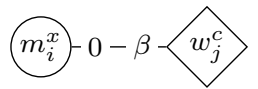

(a)

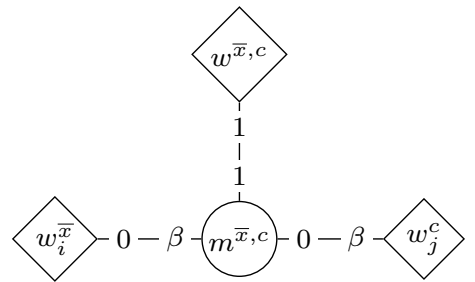

(b)

Figure 10: VC-connectors corresponding to (a) clause $c$ and positive literal $x$, and (b) clause $c$ and negative literal $\bar{x}$.

Any other pair $(m, w)$ that does not appear as an edge in the graph representation has $U(m, w)=V(m, w)=$

The instance $\mathcal{I}$ contains a variable gadget for every variable $x$, which consists of five men $m_{1}^{x}, m_{2}^{x}, e_{1}^{x}, e_{2}^{x}$, $e_{3}^{x}$, four women $w_{1}^{\bar{x}}, w_{2}^{\bar{x}}, f_{1}^{x}, f_{2}^{x}$ and the ten balanced edges $\left(e_{1}^{x}, f_{1}^{x}\right),\left(m_{1}^{x}, f_{1}^{x}\right),\left(m_{1}^{x}, w_{1}^{\bar{x}}\right),\left(e_{3}^{x}, w_{1}^{\bar{x}}\right),\left(e_{3}^{x}, w_{2}^{\bar{x}}\right)$, $\left(m_{2}^{x}, w_{2}^{\bar{x}}\right),\left(m_{2}^{x}, f_{2}^{x}\right),\left(e_{2}^{x}, f_{2}^{x}\right),\left(m_{1}^{x}, w_{2}^{\bar{x}}\right)$, and $\left(m_{2}^{x}, w_{1}^{\bar{x}}\right)$ (see Figure 9 ).

For every clause $c$, instance $\mathcal{I}$ has a clause gadget with three men $m^{c}, e_{1}^{c}, e_{2}^{c}$, three women $w_{1}^{c}, w_{2}^{c}, w_{3}^{c}$, and the nine balanced edges between them (see Figure $9 \mathrm{~b}$ ).

For every appearance of a literal in a clause, there is a variable-clause connector (or VC-connector) whose structure depends on whether it corresponds to a positive or a negative literal. In particular, for every positive literal $x$ whose $i$-th appearance $(i \in\{1,2\})$ is as the $j$-th literal $(j \in\{1,2,3\})$ of clause $c$, instance $\mathcal{I}$ has a $\mathrm{VC}$-connector that consists of a single woman-heavy edge between $m_{i}^{x}$ (from the variable gadget corresponding to variable $x$ ) and $w_{j}^{c}$ (from the clause gadget corresponding to clause $c$ ) such that $U\left(m_{i}^{x}, w_{j}^{c}\right)=0$ and $V\left(m_{i}^{x}, w_{j}^{c}\right)=\beta$ (see Figure 10 ). This edge is simultaneously the input and output edge of the VC-connector.

Similarly, for every negative literal $\bar{x}$ whose $i$-th appearance $(i \in\{1,2\})$ is as the $j$-th literal $(j \in\{1,2,3\})$ of clause $c$, instance $\mathcal{I}$ has a VC-connector that consists of man $m^{\bar{x}, c}$, woman $w^{\bar{x}, c}$, the man-heavy edge 


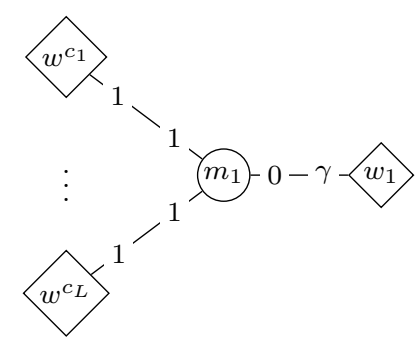

Figure 11: The accumulator. conditions

(1) $\mu\left(m_{1}^{x}, w_{1}^{\bar{x}}\right)+\mu\left(m_{1}^{x}, w_{2}^{\bar{x}}\right)+\mu\left(m_{1}^{x}, f_{1}^{x}\right) \geq 1-\varepsilon$ and $\mu\left(m_{2}^{x}, w_{1}^{\bar{x}}\right)+\mu\left(m_{2}^{x}, w_{2}^{\bar{x}}\right)+\mu\left(m_{2}^{x}, f_{2}^{x}\right) \geq 1-\varepsilon$.

(2) $\mu\left(m_{1}^{x}, w_{1}^{\bar{x}}\right)+\mu\left(m_{2}^{x}, w_{1}^{\bar{x}}\right)+\mu\left(e_{3}^{x}, w_{1}^{\bar{x}}\right) \geq 1-\varepsilon$ and $\mu\left(m_{1}^{x}, w_{2}^{\bar{x}}\right)+\mu\left(m_{2}^{x}, w_{2}^{\bar{x}}\right)+\mu\left(e_{3}^{x}, w_{2}^{\bar{x}}\right) \geq 1-\varepsilon$.

Proof. Assume otherwise that, for some $i, j \in\{1,2\}, \mu\left(m_{i}^{x}, w_{1}^{\bar{x}}\right)+\mu\left(m_{i}^{x}, w_{2}^{\bar{x}}\right)+\mu\left(m_{i}^{x}, f_{i}^{x}\right)<1-\varepsilon$ and 985 and no woman-heavy edge $\left(m, w_{j}^{\bar{x}}\right)$, both $m_{i}^{x}$ and $w_{j}^{\bar{x}}$ have utility less than $1-\varepsilon$ in $\mu$ and hence the pair $\left(m_{i}^{x}, w_{j}^{\bar{x}}\right)$ is $\varepsilon$-blocking - a contradiction. 
As before, the two conditions in the statement of Claim 5 affect the weight of the input edges of the VC-connectors that are attached to the variable gadget in any $\varepsilon$-stable fractional matching. Thus, condition

990

(1) implies that the weight assigned to each input edge of the VC-connectors that correspond to the two appearances of the positive literal $x$ in clauses must be at most $\varepsilon$. To see why, observe that these input edges are incident to nodes $m_{1}^{x}$ and $m_{2}^{x}$, and the total weight of all edges incident to each of these nodes cannot exceed 1. Similarly, condition (2) implies that the weight assigned to each input edge of the VC-connectors that correspond to the two appearances of the negative literal $\bar{x}$ in clauses must be at most $\varepsilon$.

Claim 6. Any $\varepsilon$-stable fractional matching that assigns a weight of at most $\varepsilon$ to the input edge of a VCconnector must assign a weight of at most $\beta \varepsilon$ to its output edge as well.

Proof. The claim holds trivially for VC-connectors corresponding to positive literals. Consider a VCconnector corresponding to a negative literal $\bar{x}$ and a clause $c$ containing it, and let $\zeta \leq \varepsilon$ be the weight assigned to the input edge $\left(m^{\bar{x}, c}, w_{i}^{\bar{x}}\right)$. Observe that, besides the input edge $\left(m^{\bar{x}, c}, w_{i}^{\bar{x}}\right)$, the edge $\left(m^{\bar{x}, c}, w^{\bar{x}, c}\right)$ is the only balanced or man-heavy edge that is incident to man $m^{\bar{x}, c}$ and the only balanced (or woman-heavy) edge incident to woman $w^{\bar{x}, c}$. Hence, $\varepsilon$-stability of edge $\left(m^{\bar{x}, c}, w^{\bar{x}, c}\right)$ requires a weight of at least $1-\varepsilon-\beta \zeta$ assigned to it. Then, the output edge, which is also incident to node $m^{\bar{x}, c}$, must have a weight of at most $\varepsilon+(\beta-1) \zeta \leq \beta \varepsilon$.

Claim 7. Any $\varepsilon$-stable fractional matching that assigns a weight of at most $\beta \varepsilon$ to each output edge of the $V C$-connectors corresponding to clause c must assign a weight of at most $3\left(\beta^{2}+1\right) \varepsilon$ to the CA-connector of clause $c$ as well.

Proof. Let $\ell_{1}, \ell_{2}$, and $\ell_{3}$ be the literals of clause $c$. Consider, for the sake of contradiction, an $\varepsilon$-stable fractional matching $\mu$ that assigns (1) a weight of at most $\beta \varepsilon$ to each output edge of the VC-connectors corresponding to literals $\ell_{i}$ and clause $c$, and $(2)$ a weight of more than $3\left(\beta^{2}+1\right) \varepsilon$ to edge $\left(m^{c}, w^{c}\right)$ of the CA-connector for clause $c$. Note that condition (2) implies that the total weight on the edges $\left(m^{c}, w_{1}^{c}\right)$, $\left(m^{c}, w_{2}^{c}\right)$ and $\left(m^{c}, w_{3}^{c}\right)$ is strictly smaller than $1-3\left(\beta^{2}+1\right) \varepsilon$. Since these are the only balanced or man-heavy edges incident to man $m^{c}$, the $\varepsilon$-stability of these edges is guaranteed by a utility of (at least) $1-\varepsilon$ for each of the agents $w_{1}^{c}, w_{2}^{c}$, and $w_{3}^{c}$.

Note that besides the output edges of the VC-connectors, the edges $\left(e_{1}^{c}, w_{i}^{c}\right),\left(e_{2}^{c}, w_{i}^{c}\right)$, and $\left(m^{c}, w_{i}^{c}\right)$ are the only balanced or woman-heavy edges incident to agent $w_{i}^{c}$ for all $i \in\{1,2,3\}$. Along with condition (1), this implies that the weight assigned to these three edges is at least $1-\left(\beta^{2}+1\right) \varepsilon$. Hence, the total weight on the nine edges of the clause gadget is at least $3-3\left(\beta^{2}+1\right) \varepsilon$, which, by the definition of $\beta$, is strictly more than 2 for the six edges incident to men $e_{1}^{c}$ and $e_{2}^{c}$, violating the definition of a fractional matching. 
Claim 8. Any $\varepsilon$-stable fractional matching that assigns a weight of at most $3\left(\beta^{2}+1\right) \varepsilon$ to at least two $C A$ connectors must assign a total weight of $1-\varepsilon$ to the tine edges and a weight of at most $\varepsilon$ to the edge $\left(m_{1}, w_{1}\right)$ of the accumulator.

Proof. Assume that a weight of at most $3\left(\beta^{2}+1\right) \varepsilon$ has been assigned to the edges $\left(m^{c_{1}}, w^{c_{1}}\right)$ and $\left(m^{c_{2}}, w^{c_{2}}\right)$ of the CA-connectors corresponding to some clauses $c_{1}$ and $c_{2}$. Since these are the only edges for which agents $w^{c_{1}}$ and $w^{c_{2}}$ have positive value, and there is no man-heavy edge incident to agent $m_{1}$, stability on the edges $\left(m_{1}, w^{c_{1}}\right)$ and $\left(m_{1}, w^{c_{2}}\right)$ requires that the total weight of the tine edges $\left(m_{1}, w^{c}\right)$ (for every clause c) is (at least) $1-\varepsilon$. Indeed, since by the definition of $\beta$ we have $\left(3 \beta^{2}+4\right) \varepsilon<1 / 2$, it is not possible to guarantee stability of the edges $\left(m_{1}, w^{c_{1}}\right)$ and $\left(m_{1}, w^{c_{2}}\right)$ by assigning weight at least $1-\left(3 \beta^{2}+4\right) \varepsilon$ to each of them so that both $w^{c_{1}}$ and $w^{c_{2}}$ have utility at least $1-\varepsilon$. Hence, the weight of the edge $\left(m_{1}, w_{1}\right)$ of the accumulator is at most $\varepsilon$.

\subsection{Proof of inapproximability}

Lemma 6. If formula $\phi$ is not satisfiable, then any $\varepsilon$-stable fractional matching of $\mathcal{I}$ has welfare at most $56 \beta N+\gamma \varepsilon$.

Proof. We will first show that if $\phi$ is not satisfiable, then any $\varepsilon$-stable fractional matching of $\mathcal{I}$ assigns weight at most $3\left(\beta^{2}+1\right) \varepsilon$ to at least two CA-connectors. For the sake of contradiction, consider an $\varepsilon$-stable fractional matching that assigns weight at most $3\left(\beta^{2}+1\right) \varepsilon$ to at most one CA-connector; let $c$ be the relevant clause, if such a clause exists. We will construct a truth assignment for formula $\phi$ (contradicting the assumption of the lemma) by repeating the following process for every clause $c^{\prime} \neq c$ of $\phi$. Let $\ell$ be a literal that appears in $c^{\prime}$ such that the output edge of the VC-connector, that corresponds to the appearance of $\ell$ in $c^{\prime}$, has weight greater than $\beta \varepsilon$. Recall that such a literal exists by Claim 7. We set $\ell$ to 1 (true). For every variable that has not received a value in this way, we arbitrarily set it to 1 .

The above assignment satisfies all clauses except possibly for clause $c$. Since clause $c$ is coupled with another clause $\hat{c}$ that is satisfied, it suffices to assign one of the variables appearing in $c$ the same value as its corresponding coupled variable appearing in $\hat{c}$. To show that the assignment is also valid, we need to argue that there is no variable $x$ such that both literals $x$ and $\bar{x}$ have been set to 1 . Assume otherwise that this is the case. Furthermore, assume that literal $x$ was set to 1 due to its appearance in a clause $c_{1}$, and that this is its $i_{1}$-th appearance (with $i_{1} \in\{1,2\}$ ). Also, literal $\bar{x}$ was set to 1 due to its appearance in a different clause $c_{2}$, where $\bar{x}$ makes its $i_{2}$-th appearance (again, $i_{2} \in\{1,2\}$ ). Hence, the output edge of the VC-connector that corresponds to literal $x$ and clause $c_{1}$ (respectively, the VC-connector that corresponds to literal $\bar{x}$ and clause $c_{2}$ ) has weight greater than $\beta \varepsilon$. Then, by Claim 6 , the input edges of both VC-connectors have weight greater than $\varepsilon$. As these input edges are incident to nodes $m_{i_{1}}^{x}$ and $w_{i_{2}}^{\bar{x}}$, Claim 5 yields that the total weight 
in the edges incident to some of the nodes $m_{i_{1}}^{x}$ and $w_{i_{2}}^{\bar{x}}$ is strictly higher than 1 , contradicting the definition of a fractional matching.

Since any $\varepsilon$-stable fractional matching assigns a weight of at most $3\left(\beta^{2}+1\right) \varepsilon$ to at least two CA-connectors, by Claim 8 , the contribution of the accumulator to the welfare is at most $2(1-\varepsilon)+\gamma \varepsilon(2(1-\varepsilon)$ from the tine edges plus $\gamma \varepsilon$ from the accumulator). The upper bound follows by considering the sum of valuations of all agents for edges that do not belong to the accumulator. This sum consists of

- total value of 20 for the ten balanced edges of each of the $N$ variable gadgets,

- total value of $\beta$ for the edges of each of the $2 N$ VC-connectors corresponding to a positive literal,

- total value of $2+2 \beta$ for the edges of each of the $2 N$ VC-connectors corresponding to a negative literal,

- total value of 19 for the nine balanced edges of each of the $4 N / 3$ clause gadgets and their corresponding CA-connectors.

It can be easily seen that $56 \beta N-2(1-\varepsilon)$ is a (loose) upper bound on the total value from these edges.

Lemma 7. If $\phi$ is satisfiable, then there exists an $\varepsilon$-stable fractional matching on $\mathcal{I}$ that has welfare at least $\gamma$.

Proof. Consider an assignment of boolean values to the variables that satisfies $\phi$. We construct an $\varepsilon$-stable fractional matching $\mu$ in $\mathcal{I}$ so that the contribution of the accumulator gadget to the welfare is at least $\gamma$.

Variable gadgets.. The weights on the edges of the variable gadget corresponding to variable $x$ are:

- $\mu\left(m_{1}^{x}, w_{1}^{\bar{x}}\right)=\mu\left(e_{3}^{x}, w_{1}^{\bar{x}}\right)=\mu\left(e_{3}^{x}, w_{2}^{\bar{x}}\right)=\mu\left(m_{2}^{x}, w_{2}^{\bar{x}}\right)=1 / 2, \mu\left(e_{1}^{x}, f_{1}^{x}\right)=\mu\left(e_{2}^{x}, f_{2}^{x}\right)=1$, and $\mu\left(m_{1}^{x}, f_{1}^{x}\right)=$ $\mu\left(m_{1}^{x}, w_{2}^{\bar{x}}\right)=\mu\left(m_{2}^{x}, f_{2}^{x}\right)=\mu\left(m_{2}^{x}, w_{1}^{\bar{x}}\right)=0$ if $x$ is true, and

- $\mu\left(e_{3}^{x}, w_{1}^{\bar{x}}\right)=\mu\left(e_{3}^{x}, w_{2}^{\bar{x}}\right)=1 / 2, \mu\left(m_{1}^{x}, f_{1}^{x}\right)=\mu\left(m_{2}^{x}, f_{2}^{x}\right)=1, \mu\left(m_{1}^{x}, w_{1}^{\bar{x}}\right)=\mu\left(m_{2}^{x}, w_{2}^{\bar{x}}\right)=\mu\left(m_{1}^{x}, w_{2}^{\bar{x}}\right)=$ $\mu\left(m_{2}^{x}, w_{1}^{\bar{x}}\right)=\mu\left(e_{1}^{x}, f_{1}^{x}\right)=\mu\left(e_{2}^{x}, f_{2}^{x}\right)=0$ if $x$ is false.

Clause gadgets and CA-connectors.. For every clause we select arbitrarily one of the true literals of the clause and call it active; since the assignment satisfies $\phi$, there is certainly such a literal. Consider clause $c$ and let $\ell_{i}$ (with $i \in\{1,2,3\}$ ) be its active literal; let $i_{1}$ and $i_{2}$ be the indices from $\{1,2,3\}$ than are different than $i$. We set $\mu\left(e_{1}^{c}, w_{i_{1}}^{c}\right)=\mu\left(e_{2}^{c}, w_{i_{2}}^{c}\right)=1$ and $\mu\left(e_{1}^{c}, w_{i}^{c}\right)=\mu\left(e_{1}^{c}, w_{i_{2}}^{c}\right)=\mu\left(e_{2}^{c}, w_{i}^{c}\right)=\mu\left(e_{2}^{c}, w_{i_{1}}^{c}\right)=\mu\left(m^{c}, w_{i}^{c}\right)=$ $\mu\left(m^{c}, w_{i_{1}}^{c}\right)=\mu\left(m^{c}, w_{i_{2}}^{c}\right)=0$. We also assign a weight of 1 to the CA-connector corresponding to $c$, i.e., $\mu\left(m^{c}, w^{c}\right)=1$. 
VC-connectors.. For every non-active VC-connector, we set the weight of its balanced edge, if it exists, to 1 and the weight of the remaining edges to 0 . For every active VC-connector corresponding to the

$i$-th appearance of the positive literal $x$ as the $j$-th literal of clause $c(i \in\{1,2\}, j \in\{1,2,3\})$, we set $\mu\left(m_{i}^{x}, w_{j}^{c}\right)=1 / 2$.

For every active $\mathrm{VC}$-connector corresponding to the $i$-th appearance of the negative literal $x$ as the $j$ th literal of clause $c(i \in\{1,2\}, j \in\{1,2,3\})$, we set the weights of its edges as follows: $\mu\left(m^{\bar{x}, c}, w_{i}^{\bar{x}}\right)=$ $\mu\left(m^{\bar{x}, c}, w_{j}^{c}\right)=1 / 2$ and $\mu\left(m^{\bar{x}, c}, w^{\bar{x}, c}\right)=0$.

Accumulator.. We set $\mu\left(m_{1}, w^{c}\right)=0$ for every tine edge $\left(m_{1}, w^{c}\right)$ of the accumulator. Furthermore, we set $\mu\left(m_{1}, w_{1}\right)=1$. So, the contribution of the accumulator to the social welfare is $\gamma$, as desired.

It can be easily verified that the total weight of the edges that are incident to any node is at most 1 . Hence, $\mu$ is a valid fractional matching. Regarding stability, it suffices to verify that either the man or the woman of a balanced pair has a utility of at least $1-\varepsilon$. Note that we only need to check for the balanced edges, as the man- or woman-heavy edges and the remaining pairs do not impose any constraints on stability. For the balanced edges, stability is established by the following series of observations (we will use the term 'stabilized by' to denote that an agent's utility is at least $1-\varepsilon)$ : The variable gadget for the variable $x$ (Figure 9a) is stabilized by the agents $f_{1}^{x}, f_{2}^{x}, e_{3}^{x}$ along with $m_{1}^{x}, m_{2}^{x}$ (if $x$ is true) or $w_{1}^{\bar{x}}, w_{2}^{\bar{x}}$ (if $x$ is false). The clause gadget for clause $c$ (Figure 9p) with active index $i$ (and non-active indices $i_{1}$ and $i_{2}$ ) is stabilized by the agents $e_{1}^{c}, e_{2}^{c}, w_{i}^{c}, w_{i_{1}}^{c}, w_{i_{2}}^{c}$; in particular, the edge $\left(m^{c}, w_{i}^{c}\right)$ is stabilized by $w_{i}^{c}$ because an active literal triggers the woman-heavy edge in the VC-connector. A VC connector is stabilized by $m^{\bar{x}, c}$ (Figure 10p). Finally, the tine edges in the accumulator (Figure 11) are stabilized by $w^{c_{1}}, \ldots, w^{c_{L}}$ (because we trigger the CA-connector). Overall, $\mu$ is a feasible stable fractional matching.

We are ready to prove Theorem 8 . We select a value of $\gamma$ such that $\gamma \geq \frac{56 \beta N(1 / \varepsilon-\delta)}{\varepsilon \delta}$. By Lemma 6 , the welfare of $\mu$ if $\phi$ was not satisfiable would be at most

$$
56 \beta N+\gamma \varepsilon \leq \frac{\gamma \varepsilon \delta}{1 / \varepsilon-\delta}+\gamma \varepsilon=\frac{\gamma}{1 / \varepsilon-\delta} .
$$

By Lemma 7, we have that the welfare of $\mu$ if $\phi$ was not satisfiable would be at least $1 / \varepsilon-\delta$ times smaller than the welfare $\mathcal{I}$ could have if $\phi$ was satisfiable.

\section{Approximation Algorithm for $\frac{1}{2}$-stability}

We will now provide a polynomial-time algorithm that computes a $\frac{1}{2}$-stable fractional matching with welfare at least that of an optimal (exactly) stable fractional matching. Notice that unlike Theorems 5 and 6 , where the quality of the computed matching is compared to the optimal matching $\mu^{\mathrm{opt}}$, the guarantee in Theorem 9 is considerably weaker. 
Theorem 9. Let $\mathcal{I}$ be an SMC instance and $\mu^{*}$ be an optimal stable fractional matching for $\mathcal{I}$. Then, a $\frac{1}{2}$-stable fractional matching $\mu$ that satisfies $\mathcal{W}(\mu) \geq \mathcal{W}\left(\mu^{*}\right)$ can be computed in polynomial time.

Proof. Consider the mixed integer linear program (OPT-Stab) from Section 2.1 for finding an optimal stable fractional matching for $\mathcal{I}$. Relaxing the integrality constraint $[8]$ to $y(m, w) \in[0,1]$ results in a linear program. Since a stable fractional matching always exists (see Proposition 1), this relaxation is feasible. Let $\mu$ be a solution of the relaxed program. Since $\max \{y(m, w), 1-y(m, w)\} \geq \frac{1}{2}$, we have that for every man-woman pair $(m, w) \in M \times W$, either $u_{m} \geq \frac{1}{2} U(m, w)$ or $v_{w} \geq \frac{1}{2} V(m, w)$, implying that $\mu$ is $\frac{1}{2}$-stable. It is also clear that $\mathcal{W}(\mu) \geq \mathcal{W}\left(\mu^{*}\right)$ since $\mu^{*}$ satisfies (OPT-Stab). 